MATHEMATICS OF COMPUTATION

Volume 77, Number 262, April 2008, Pages 699-730

S 0025-5718(07)02045-5

Article electronically published on September 6, 2007

\title{
A DISCONTINUOUS GALERKIN FINITE ELEMENT METHOD FOR TIME DEPENDENT PARTIAL DIFFERENTIAL EQUATIONS WITH HIGHER ORDER DERIVATIVES
}

\author{
YINGDA CHENG AND CHI-WANG SHU
}

\begin{abstract}
In this paper, we develop a new discontinuous Galerkin (DG) finite element method for solving time dependent partial differential equations (PDEs) with higher order spatial derivatives. Unlike the traditional local discontinuous Galerkin (LDG) method, the method in this paper can be applied without introducing any auxiliary variables or rewriting the original equation into a larger system. Stability is ensured by a careful choice of interface numerical fluxes. The method can be designed for quite general nonlinear PDEs and we prove stability and give error estimates for a few representative classes of PDEs up to fifth order. Numerical examples show that our scheme attains the optimal $(k+1)$-th order of accuracy when using piecewise $k$-th degree polynomials, under the condition that $k+1$ is greater than or equal to the order of the equation.
\end{abstract}

\section{Introduction}

In this paper, we develop a new discontinuous Galerkin (DG) method to solve time dependent partial differential equations with higher order spatial derivatives. We describe the designing principle of our new DG scheme through a few representative examples of such equations including the generalized KdV equation

$$
u_{t}+f(u)_{x}+\sigma u_{x x x}=0,
$$

the convection diffusion equation

$$
u_{t}+f(u)_{x}-\left(a(u) u_{x}\right)_{x}=0
$$

with $a(u) \geq 0$, a type of time dependent biharmonic equation

$$
u_{t}+f(u)_{x}+\sigma u_{x x x x}=0
$$

with $\sigma>0$, a type of nonlinear equation with fifth order derivative

$$
u_{t}+f(u)_{x}+\sigma u_{x x x x x}=0,
$$

and the fully nonlinear fifth order $K(n, n, n)$ equation

$$
u_{t}+\left(u^{n}\right)_{x}+\left(u^{n}\right)_{x x x}+\left(u^{n}\right)_{x x x x x}=0 .
$$

Received by the editor August 25, 2006 and, in revised form, February 17, 2007.

2000 Mathematics Subject Classification. Primary 65M60.

Key words and phrases. Discontinuous Galerkin method, partial differential equations with higher order derivatives, stability, error estimate, high order accuracy.

This research was supported in part by ARO grant W911NF-04-1-0291, NSF grant DMS0510345 and AFOSR grant FA9550-05-1-0123.

(C)2007 American Mathematical Society Reverts to public domain 28 years from publication 
We concentrate our attention in one dimension for this paper, however, the method as well as its stability analysis can be easily generalized to multi-dimensional cases.

The type of DG method that we will discuss here is a class of finite element methods originally devised to solve hyperbolic conservation laws containing only first order spatial derivatives, e.g. [11, 10, 9, 8, 12. Using completely discontinuous polynomial space for both the test and trial functions in the spatial variables and coupled with explicit and nonlinearly stable high order Runge-Kutta time discretization, the method has the advantage of flexibility for arbitrarily unstructured meshes, with a compact stencil, and with the ability to easily accommodate arbitrary $h-p$ adaptivity.

The DG method was later generalized to the local DG (LDG) method by Cockburn and Shu to solve the convection diffusion equation [13. Their work was motivated by the successful numerical experiments of Bassi and Rebay [ 5 ] for the compressible Navier-Stokes equations. Later, Yan and Shu developed a LDG method for a general $\mathrm{KdV}$ type equation containing third order derivatives in [27], and they generalized the LDG method to PDEs with fourth and fifth spatial derivatives in [28. Levy, Shu and Yan [17] developed LDG methods for nonlinear dispersive equations that have compactly supported traveling wave solutions, the so-called "compactons". More recently, Xu and Shu further generalized the LDG method to solve a series of nonlinear wave equations $22,23,24,25$.

The idea of the LDG method is to introduce new auxiliary variables and rewrite the original equation into several first order equations. By carefully choosing the numerical fluxes, the LDG scheme is proven to be stable and high order accurate. The LDG method can also retain the flexibility of the DG method since the auxiliary variables can be locally eliminated, hence the word local in LDG. However, practitioners are sometimes unhappy with these auxiliary variables, since they may increase the complexity and computational cost of the method, and expand the effective stencil of the method after the elimination of the auxiliary variables. An alternative method for solving second order convection diffusion equations is the DG method of Baumann and Oden [6; see also 19. This method does not need the introduction of auxiliary variables, relying instead on penalty terms at cell boundaries to achieve stability. However, the method does not achieve the optimal $(k+1)$-th order of accuracy when piecewise polynomials of an even degree $k$ is used. Also, it does not seem straightforward to generalize the method to nonlinear PDEs containing higher spatial derivatives. Another class of related methods, mostly for elliptic problems with even-order leading derivatives and without time, is the class of interior penalty (IP) methods, first discussed by Baker in [4]; see also [2, 3]. The IP methods rely on penalty terms, typically proportional to the jumps of the solution, added at all interior cell interfaces to stabilize the methods. Our method in this paper, when suitably rewritten, can be cast into the unified framework [3] to which the IP methods also belong. Advantages of our approach, through the design of numerical fluxes, include its automatic local conservation, and its ability to design stable DG methods for wave type PDEs with odd-order leading derivatives such as the KdV equations. More recently, van Leer and Nomura 21] and Gassner et al. [15] proposed new DG formulations for the diffusion equations. They use twice the integration by parts for the diffusion term, and either an $L^{2}$ projection of the discontinuous piecewise polynomial over two neighboring cells into a continuous, single polynomial, or a suitable Riemann solver for the diffusion equation, that is, 
exact solutions for the diffusion equation with a step function Riemann initial data, to provide interface values of the solution derivative resulting from the integration by parts. The DG schemes in 21] and [15] do not need to use the auxiliary variables as in LDG method, however, the $L^{2}$ projection procedure might be cumbersome for arbitrary triangulations in multi-dimensions, especially for non-conforming meshes with hanging nodes, while the Riemann solver based approach might be difficult to be generalized to equations containing higher order spatial derivatives. Stability and convergence of the schemes are not proven in 21] and [15. In 11, Adjerid and Temimi introduced a DG method for solving high order ordinary differential equations (ODEs). Their method relies on a repeated integration by parts and taking the numerical traces (fluxes) always from the left (the side with the smaller time). The objective of our study is to develop a framework of designing DG schemes that will work for general time dependent PDEs with higher order spatial derivatives, by carefully choosing the numerical fluxes resulting from integration by parts to ensure provable stability. Similar to [21, 15, 1], we also rely on repeated integration by parts; and similar to [1, we also rely on the choice of numerical fluxes at the cell interfaces. However, since we are dealing with PDEs, with possibly nonlinear higher order derivative terms, the simple choice of taking the numerical fluxes always from the left does not work and we have to carefully design the numerical fluxes for different PDEs to ensure stability. The DG schemes discussed in this paper are more compact than LDG schemes and are simpler in formulation and coding.

The organization of the paper is as follows. In Section 2, we introduce notations and some auxiliary results that will be used later in the paper. In Section 3, the scheme for the generalized KdV equations is discussed. Stability, error estimates and numerical examples will be given. In Section 4, we follow the lines of Section 3 and consider the convection diffusion equation. In Section 5, we generalize our scheme to higher order equations. Concluding remarks and comments for future work are provided in Section 6. Finally, in the Appendix we provide proofs for some of the more technical results of the error estimates.

\section{Notations AND AUXiliary RESUlts}

In this section, we introduce notations and some auxiliary results that will be used later.

2.1. Basic notations. In this paper, we will follow the usual notation of the DG method. For a given interval $I=[a, b]$, we divide it into $N$ cells as follows:

$$
a=x_{\frac{1}{2}}<x_{\frac{3}{2}}<\ldots<x_{N+\frac{1}{2}}=b .
$$

We denote

$$
I_{j}=\left(x_{j-\frac{1}{2}}, x_{j+\frac{1}{2}}\right), \quad x_{j}=\frac{1}{2}\left(x_{j-\frac{1}{2}}+x_{j+\frac{1}{2}}\right)
$$

and

$$
h_{j}=x_{j+\frac{1}{2}}-x_{j-\frac{1}{2}}, \quad h=\max _{j} h_{j} .
$$

We also assume the mesh is regular, i.e., the ratio between the maximum and minimum mesh sizes shall stay bounded during mesh refinements. We define the 
approximation space as

$$
V_{h}^{k}=\left\{v_{h}:\left.\left(v_{h}\right)\right|_{I_{j}} \in P^{k}\left(I_{j}\right), j=1, \ldots, N\right\} .
$$

Here $P^{k}\left(I_{j}\right)$ denotes the set of all polynomials of degree at most $k$ on $I_{j}$. For a function $v_{h} \in V_{h}^{k}$, we use $\left(v_{h}\right)_{j+\frac{1}{2}}^{-}$and $\left(v_{h}\right)_{j+\frac{1}{2}}^{+}$to refer to the value of $v_{h}$ at $x_{j+\frac{1}{2}}$ from the left cell $I_{j}$ and the right cell $I_{j+1}$, respectively. $\left[v_{h}\right]$ is used to denote $v_{h}^{+}-v_{h}^{-}$, i.e. the jump of $v_{h}$ at cell interfaces. $\bar{v}_{h}=\frac{1}{2}\left(v_{h}^{-}+v_{h}^{+}\right)$denotes the average of the left and right boundary values.

2.2. Notations for different constants. For notations of different constants we will follow [29] and 26]. By $C$, we refer to a positive constant that is independent of the mesh size $h$, but it may depend on other parameters of the problem. Especially, to emphasize the nonlinearity of the flux $f(u)$, we use $C_{\star}$ to denote a non-negative constant depending on the maximum of $\left|f^{\prime \prime}\right|$ and $\left|f^{\prime \prime \prime}\right| . C_{\star}=0$ for linear fluxes $f(u)=a u$ with a constant $a$.

2.3. Numerical flux. In this paper, we will be using monotone numerical fluxes $\hat{f}\left(w^{-}, w^{+}\right)$. It satisfies the following conditions:

(1) It is locally Lipschitz continuous.

(2) It is consistent with the flux $f(w)$, i.e., $\hat{f}(w, w)=f(w)$.

(3) It is a nondecreasing function of its first argument, and a nonincreasing function of its second argument.

2.4. Projection properties. For the error estimates, we will be using various kinds of projection $P_{h}$ into $V_{h}^{k}$. For example, we can choose $P_{h}$ such that for any $u, P_{h} u$ satisfies

$$
\int_{I_{j}} u v_{h} d x=\int_{I_{j}} P_{h} u v_{h} d x
$$

for any $v_{h} \in V_{h}^{k-3}$ and

$$
P_{h} u^{+}=u^{+}, \quad\left(P_{h} u\right)_{x}^{+}=u_{x}^{+}, \quad\left(P_{h} u\right)_{x x}^{-}=u_{x x}^{-}
$$

at all $x_{j+1 / 2}$. We will use different projection $P_{h}$ according to the need in each proof. For all these projections, the following inequality holds [7]:

$$
\left\|w^{e}\right\|+h\left\|w^{e}\right\|_{\infty}+h^{\frac{1}{2}}\left\|w^{e}\right\|_{\Gamma_{h}} \leq C h^{k+1}
$$

where $w^{e}=P_{h} w-w, \Gamma_{h}$ denotes the set of boundary points of all elements $I_{j}$, and the constant $C$ depends on $k$ and the standard Sobolev $k+1$ semi-norm $|u|_{k+1}$ of the smooth function $w$. Here and below, an unmarked norm $\|\cdot\|$ denotes the $L^{2}$ norm.

2.5. Inverse properties. For any function $w_{h} \in V_{h}^{k}$, the following inequalities hold [7:

$$
\text { (i) }\left\|\left(w_{h}\right)_{x}\right\| \leq C h^{-1}\left\|w_{h}\right\| \text {, }
$$$$
\text { (ii) }\left\|w_{h}\right\|_{\Gamma_{h}} \leq C h^{-\frac{1}{2}}\left\|w_{h}\right\| \text {, }
$$$$
\text { (iii) }\left\|w_{h}\right\|_{\infty} \leq C h^{-\frac{1}{2}}\left\|w_{h}\right\| \text {. }
$$ 
2.6. Time discretizations. In this paper, we will be using two types of time discretizations. One of them is the total variation diminishing (TVD) high-order Runge-Kutta method [20. For the method of lines ODE

$$
\left(u_{h}\right)_{t}=L\left(u_{h}\right) \text {, }
$$

the third order TVD Runge-Kutta method that we use in this paper is given by

$$
\begin{aligned}
u_{h}^{(1)} & =u_{h}^{n}+\triangle t L\left(u_{h}^{n}\right), \\
u_{h}^{(2)} & =\frac{3}{4} u_{h}^{n}+\frac{1}{4} u_{h}^{(1)}+\frac{1}{4} \triangle t L\left(u_{h}^{(1)}\right), \\
u_{h}^{n+1} & =\frac{1}{3} u_{h}^{n}+\frac{2}{3} u_{h}^{(2)}+\frac{2}{3} \triangle t L\left(u_{h}^{(2)}\right) .
\end{aligned}
$$

We will also use the backward Euler scheme as

$$
u_{h}^{n+1}=u_{h}^{n}+\triangle t L\left(u_{h}^{n+1}\right) .
$$

For PDEs containing high order spatial derivatives, explicit time discretization such as (2.8) is subject to severe time step restriction for stability. More efficient time stepping techniques such as the exponential time differencing (ETD) method [14] or preconditioned implicit methods should be used. We do not pursue this issue since the focus of this paper is on the DG spatial discretization.

\section{The KdV EQUation}

We first consider the one dimensional generalized KdV equation given by

$$
u_{t}+f(u)_{x}+\sigma u_{x x x}=0
$$

where $\sigma$ is a given constant, to demonstrate the basic ideas of our DG scheme.

We propose our scheme as follows: find $u_{h} \in V_{h}^{k}$, such that

$$
\begin{aligned}
& \int_{I_{j}}\left(u_{h}\right)_{t} v_{h} d x-\int_{I_{j}} f\left(u_{h}\right)\left(v_{h}\right)_{x} d x-\sigma \int_{I_{j}} u_{h}\left(v_{h}\right)_{x x x} d x \\
& +\left(\hat{f}\left(u_{h}^{-}, u_{h}^{+}\right) v_{h}^{-}\right)_{j+\frac{1}{2}}-\left(\hat{f}\left(u_{h}^{-}, u_{h}^{+}\right) v_{h}^{+}\right)_{j-\frac{1}{2}}+\sigma\left(\hat{u_{h}}\left(v_{h}\right)_{x x}^{-}\right)_{j+\frac{1}{2}} \\
& -\sigma\left(\hat{u_{h}}\left(v_{h}\right)_{x x}^{+}\right)_{j-\frac{1}{2}}-\sigma\left(\left(\tilde{u}_{h}\right)_{x}\left(v_{h}\right)_{x}^{-}\right)_{j+\frac{1}{2}}+\sigma\left(\left(\tilde{u}_{h}\right)_{x}\left(v_{h}\right)_{x}^{+}\right)_{j-\frac{1}{2}} \\
& +\sigma\left(\left(\check{u_{h}}\right)_{x x} v_{h}^{-}\right)_{j+\frac{1}{2}}-\sigma\left(\left(\check{u_{h}}\right)_{x x} v_{h}^{+}\right)_{j-\frac{1}{2}}=0
\end{aligned}
$$

holds for any $v_{h} \in V_{h}^{k}$ and $j=1, \ldots, N$. Here $\hat{f}\left(u_{h}^{-}, u_{h}^{+}\right), \hat{u_{h}},\left(\tilde{u_{h}}\right)_{x}$ and $\left(\tilde{u_{h}}\right)_{x x}$ are numerical fluxes. The terms involving these fluxes appear from repeated integration by parts, and a suitable choice for these numerical fluxes is the key ingredient for the stability of the DG scheme. The flux $\hat{f}\left(u_{h}^{-}, u_{h}^{+}\right)$is a monotone flux as described in Section 2.3. If $\sigma$ is positive, then we can take either of the following two choices for the other three fluxes

$$
\hat{u_{h}}=u_{h}^{-}, \quad\left(\tilde{u_{h}}\right)_{x}=\left(u_{h}\right)_{x}^{+}, \quad\left(\tilde{u_{h}}\right)_{x x}=\left(u_{h}\right)_{x x}^{+}
$$

or

$$
\hat{u_{h}}=u_{h}^{+}, \quad\left(\tilde{u_{h}}\right)_{x}=\left(u_{h}\right)_{x}^{+}, \quad\left(\check{u_{h}}\right)_{x x}=\left(u_{h}\right)_{x x}^{-} .
$$

It is crucial that we take $\left(\tilde{u_{h}}\right)_{x}=\left(u_{h}\right)_{x}^{+}$and $\hat{u_{h}},\left(\tilde{u_{h}}\right)_{x x}$ from the opposite directions. If $\sigma$ is negative, we can take

$$
\hat{u_{h}}=u_{h}^{+}, \quad\left(\tilde{u_{h}}\right)_{x}=\left(u_{h}\right)_{x}^{-}, \quad\left(\check{u_{h}}\right)_{x x}=\left(u_{h}\right)_{x x}^{-}
$$


or

$$
\hat{u_{h}}=u_{h}^{-}, \quad\left(\tilde{u_{h}}\right)_{x}=\left(u_{h}\right)_{x}^{-}, \quad\left(\check{u_{h}}\right)_{x x}=\left(u_{h}\right)_{x x}^{+} .
$$

The above choice of the numerical fluxes are for the periodic boundary condition, which is the only type of boundary conditions considered in this paper. For general boundary conditions, the choice of the numerical fluxes should be adjusted at the boundary; see for example [18. For simplicity of discussion, we will only consider the case of $\sigma$ being positive from now on. We note that the choice of numerical fluxes follows the same principle as those for the LDG method in [27].

3.1. Stability analysis. In this subsection, we will examine the stability property of the scheme we just proposed. As mentioned before, for simplicity we will only consider periodic boundary conditions.

Theorem 3.1. Our numerical scheme (3.2) with the flux choice (3.3) or (3.4) is $L^{2}$ stable, i.e.

$$
\left\|u_{h}(t)\right\| \leq\left\|u_{h}(0)\right\| .
$$

Proof. Let $F(u)=\int f(u) d u$. In (3.2), we let $v_{h}=u_{h}$ and sum over $j$ to obtain

$$
\frac{d}{d t} \frac{1}{2} \int_{I} u_{h}^{2} d x+\sum_{j=1}^{N} \Theta_{j-\frac{1}{2}}=0
$$

where $\Theta_{j-\frac{1}{2}}=\left(\left[F\left(u_{h}\right)\right]-\hat{f}\left(u_{h}^{-}, u_{h}^{+}\right)\left[u_{h}\right]+\frac{\sigma}{2}\left[\left(u_{h}\right)_{x}\right]^{2}\right)_{j-\frac{1}{2}}$. Equation (3.7) is true for both of our flux choices (3.3) and (3.4). It is now easy to show that $\Theta_{j-\frac{1}{2}} \geq 0$ following the proof of the cell entropy inequality in [16], using the fact that $\hat{f}$ is a monotone flux. We discuss the following two possible cases.

(1) $u_{h}^{+} \geq u_{h}^{-}$, then $\left[F\left(u_{h}\right)\right]=f(\xi)\left[u_{h}\right]$, with $\xi \in\left(u_{h}^{-}, u_{h}^{+}\right)$. From the property of a monotone flux, $f(\xi)=\hat{f}(\xi, \xi) \geq \hat{f}\left(u_{h}^{-}, u_{h}^{+}\right)$, thus $\left[F\left(u_{h}\right)\right]-$ $\hat{f}\left(u_{h}^{-}, u_{h}^{+}\right)\left[u_{h}\right] \geq 0$.

(2) $u_{h}^{+}<u_{h}^{-}$, then $\left[F\left(u_{h}\right)\right]=f(\xi)\left[u_{h}\right]$, with $\xi \in\left(u_{h}^{+}, u_{h}^{-}\right)$. From the property of a monotone flux, $f(\xi)=\hat{f}(\xi, \xi) \leq \hat{f}\left(u_{h}^{-}, u_{h}^{+}\right)$, thus $\left[F\left(u_{h}\right)\right]-$ $\hat{f}\left(u_{h}^{-}, u_{h}^{+}\right)\left[u_{h}\right] \geq 0$.

In both cases, we have $\left[F\left(u_{h}\right)\right]-\hat{f}\left(u_{h}^{-}, u_{h}^{+}\right)\left[u_{h}\right] \geq 0$, hence $\Theta_{j-\frac{1}{2}} \geq 0$, which finishes the proof.

3.2. Error estimates. In this subsection, we state the error estimates of our scheme. The proof of these results is rather technical and is therefore left to the Appendix.

Theorem 3.2. Let $u$ be the exact solution of the equation (3.1), which is sufficiently smooth with bounded derivatives, and assume $f \in C^{3}$. Let $u_{h}$ be the numerical solution of (3.2) with either (3.3) or (3.4) as the numerical fluxes. If we impose a periodic boundary condition, and use $V_{h}^{k}$ space with $k \geq 3$, then we have the following error estimate:

$$
\left\|u_{h}(t)-u(t)\right\| \leq C h^{k}
$$

where the constant $C$ depends on $k, t,\|u\|_{k+1}$ and the bounds on the derivatives $\left|f^{(m)}\right|, m=1,2,3$, but not on $h$. Here $\|u\|_{k+1}$ is the maximum over time in $[0, t]$ of the standard Sobolev $k+1$ norm in space. 
Proof. The proof of this theorem is provided in the Appendix; see Section A.2.

Remark. Notice that the error estimate (3.8) is sub-optimal. If $f(u)=0$, then the result of Theorem 3.2 can be improved to the optimal $O\left(h^{k+1}\right)$ error estimate.

Theorem 3.2 only deals with the case of $k \geq 3$. If $k=2$, the following theorem gives a more sub-optimal error estimate.

Theorem 3.3. Under the same condition as that of Theorem 3.2, if $k=2$, then we have the following error estimate:

$$
\left\|u_{h}(t)-u(t)\right\| \leq C h^{\frac{3}{2}}
$$

where the constant $C$ depends on $t,\|u\|_{k+1}$ and the bounds on the derivatives $\left|f^{(m)}\right|$, $m=1,2,3$, but not on $h$.

Proof. The proof of this theorem is provided in the Appendix; see Section A.3.

Remark. When $k<2$, numerical experiments in the following subsection show that our scheme is not consistent.

3.3. Numerical examples. In this subsection, we give numerical examples to demonstrate the performance of our scheme.

Example 3.3.1. We solve the linear third order equation given by

$$
\left\{\begin{array}{l}
u_{t}+u_{x x x}=0 \\
u(x, 0)=\sin (x) \\
u(0, t)=u(2 \pi, t) .
\end{array}\right.
$$

The exact solution is

$$
u(x, t)=\sin (x+t) .
$$

We use the third order TVD Runge-Kutta time discretization (2.8) with the time step $\triangle t=C F L h^{3}$. The optimal CFL number can be obtained by a standard von Neumann analysis. Here we simply choose a CFL number by numerical experiments to make the scheme stable. Our computation is based on the flux choice (3.4). The errors and numerical order of accuracy for $P^{k}$ elements with $1 \leq k \leq 3$ are listed in Table 3.1. We observe that our scheme is not consistent for $P^{1}$ polynomials, while optimal $(k+1)$-th order of accuracy is achieved for $k \geq 2$. However, we remark that, comparing with the LDG result in 27, for the same mesh, our DG scheme has larger errors than the LDG scheme, although both achieve the optimal order of accuracy.

Example 3.3.2. We solve the linear $\mathrm{KdV}$ equation given by

$$
\left\{\begin{array}{l}
u_{t}-u_{x}+u_{x x x}=0 \\
u(x, 0)=\sin (x) \\
u(0, t)=u(2 \pi, t)
\end{array}\right.
$$

The exact solution is

$$
u(x, t)=\sin (x+2 t) .
$$

We still use (3.4) as our flux choice and take the upwind flux for the first order convection term, i.e. $f\left(u_{h}^{-}, u_{h}^{+}\right)=-u_{h}^{+}$. Table 3.2 shows that our scheme gives the optimal $(k+1)$-th order of accuracy when $k \geq 2$. For $P^{0}$ and $P^{1}$, the scheme is not consistent. 
TABLE 3.1. Errors and numerical orders of accuracy for Example 3.3.1 when using $P^{k}$ polynomials and Runge-Kutta third order time discretization on a uniform mesh of $N$ cells. Final time $T=1$.

\begin{tabular}{|c|c|c|c|c|c|c|c|c|}
\hline$k$ & $C F L$ & $N$ & $L^{1}$ error & order & $L^{2}$ error & order & $L^{\infty}$ error & order \\
\hline \multirow{3}{*}{1} & \multirow{3}{*}{0.01} & 10 & $0.60 \mathrm{E}+00$ & - & $0.68 \mathrm{E}+00$ & - & $0.11 \mathrm{E}+01$ & - \\
\hline & & 20 & $\overline{0.61 \mathrm{E}+00}$ & -0.02 & $0.68 \mathrm{E}+00$ & 0.00 & $0.11 \mathrm{E}+01$ & 0.08 \\
\hline & & 40 & $0.61 \mathrm{E}+00$ & -0.01 & $0.68 \mathrm{E}+00$ & 0.00 & $0.10 \mathrm{E}+01$ & 0.08 \\
\hline \multirow{4}{*}{2} & \multirow{4}{*}{0.002} & 10 & $0.24 \mathrm{E}-01$ & - & $0.27 \mathrm{E}-01$ & - & $0.59 \mathrm{E}-01$ & - \\
\hline & & 20 & $0.31 \mathrm{E}-02$ & 2.96 & $0.35 \mathrm{E}-02$ & 2.96 & $0.74 \mathrm{E}-02$ & 3.00 \\
\hline & & 40 & $0.39 \mathrm{E}-03$ & 2.99 & $0.44 \mathrm{E}-03$ & 2.99 & $0.91 \mathrm{E}-03$ & 3.03 \\
\hline & & 80 & $0.49 \mathrm{E}-04$ & 3.00 & $0.55 \mathrm{E}-04$ & 3.00 & $\overline{0.11 \mathrm{E}-03}$ & 3.02 \\
\hline \multirow{4}{*}{3} & \multirow{4}{*}{0.001} & 10 & $0.24 \mathrm{E}-03$ & - & $0.32 \mathrm{E}-03$ & - & $0.10 \mathrm{E}-02$ & - \\
\hline & & 20 & $0.15 \mathrm{E}-04$ & 4.03 & $0.20 \mathrm{E}-04$ & 3.99 & $0.75 \mathrm{E}-04$ & 3.92 \\
\hline & & 40 & $0.91 \mathrm{E}-06$ & 4.02 & $0.12 \mathrm{E}-05$ & 4.00 & $0.43 \mathrm{E}-05$ & 3.98 \\
\hline & & 80 & $0.56 \mathrm{E}-07$ & 4.01 & $0.78 \mathrm{E}-07$ & 4.00 & $0.27 \mathrm{E}-06$ & 3.99 \\
\hline
\end{tabular}

TABLE 3.2. Errors and numerical orders of accuracy for Example 3.3.2 when using $P^{k}$ polynomials and Runge-Kutta third order time discretization on a uniform mesh of $N$ cells. Final time $T=1$.

\begin{tabular}{|c|c|c|cc|cc|cc|}
\hline$k$ & $C F L$ & $N$ & $L^{1}$ error & order & $L^{2}$ error & order & $L^{\infty}$ error & order \\
\hline \multirow{4}{*}{2} & \multirow{3}{*}{0.001} & 10 & $0.23 \mathrm{E}-01$ & - & $0.27 \mathrm{E}-01$ & - & $0.58 \mathrm{E}-01$ & - \\
\cline { 3 - 9 } & 20 & $0.31 \mathrm{E}-02$ & 2.90 & $0.35 \mathrm{E}-02$ & 2.93 & $0.73 \mathrm{E}-02$ & 2.98 \\
\cline { 3 - 9 } & & 40 & $0.39 \mathrm{E}-03$ & 2.98 & $0.44 \mathrm{E}-03$ & 2.99 & $0.90 \mathrm{E}-03$ & 3.02 \\
\hline & 80 & $0.49 \mathrm{E}-04$ & 2.99 & $0.55 \mathrm{E}-04$ & 3.00 & $0.11 \mathrm{E}-03$ & 3.01 \\
\hline \multirow{4}{*}{3} & \multirow{3}{*}{0.001} & 10 & $0.24 \mathrm{E}-03$ & - & $0.32 \mathrm{E}-03$ & - & $0.10 \mathrm{E}-02$ & - \\
\cline { 3 - 9 } & 20 & $0.15 \mathrm{E}-04$ & 4.04 & $0.20 \mathrm{E}-04$ & 3.99 & $0.68 \mathrm{E}-04$ & 3.92 \\
\cline { 3 - 9 } & & 40 & $0.91 \mathrm{E}-06$ & 4.02 & $0.12 \mathrm{E}-05$ & 4.00 & $0.43 \mathrm{E}-05$ & 3.98 \\
\cline { 3 - 8 } & & 0.5 & $0.56 \mathrm{E}-07$ & 4.01 & $0.78 \mathrm{E}-07$ & 4.00 & $0.27 \mathrm{E}-06$ & 3.99 \\
\hline
\end{tabular}

Example 3.3.3. We solve the classical soliton solution of the nonlinear KdV equation

$$
u_{t}-3\left(u^{2}\right)_{x}+u_{x x x}=0
$$

on the domain $[-10,12]$. The initial condition is

$$
u(x, 0)=-2 \operatorname{sech}^{2}(x) .
$$

The exact solution is

$$
u(x, t)=-2 \operatorname{sech}^{2}(x-4 t) .
$$

This example was also tested in [27. We impose the boundary condition

$$
u(-10, t)=g_{1}(t), \quad u_{x}(12, t)=g_{2}(t), \quad u_{x x}(12, t)=g_{3}(t)
$$

where $g_{i}(t)$ corresponds to the data from the exact solution, and we use the upwind flux for the first order convection term and (3.3) as our flux choice. Table 3.3 contains the numerical error and order of accuracy for $2 \leq k \leq 5$. It seems that the optimal $(k+1)$-th order of accuracy is achieved for $k \geq 3$, while for $k=2$ the error seems to be half an order lower than the optimal order 3 . 
TABLE 3.3. Errors and numerical orders of accuracy for Example 3.3.3 when using $P^{k}$ polynomials and Runge-Kutta third order time discretization on a uniform mesh of $N$ cells. Final time $T=$ 0.1 .

\begin{tabular}{|c|c|c|c|c|c|c|c|c|}
\hline$k$ & $C F L$ & $N$ & $L^{1}$ error & order & $L^{2}$ error & order & $L^{\infty}$ error & order \\
\hline \multirow{4}{*}{2} & \multirow{4}{*}{0.0001} & 20 & $0.20 \mathrm{E}-01$ & - & $0.33 \mathrm{E}-01$ & - & $0.11 \mathrm{E}+00$ & - \\
\hline & & 40 & $0.89 \mathrm{E}-02$ & 1.25 & $0.22 \mathrm{E}-01$ & 0.60 & $0.12 \mathrm{E}+00$ & -0.08 \\
\hline & & 80 & $0.26 \mathrm{E}-02$ & 1.75 & $0.61 \mathrm{E}-02$ & 1.86 & $0.28 \mathrm{E}-01$ & 2.12 \\
\hline & & 160 & $0.46 \mathrm{E}-03$ & 2.51 & $0.10 \mathrm{E}-02$ & 2.60 & $0.50 \mathrm{E}-02$ & 2.47 \\
\hline \multirow{4}{*}{3} & \multirow{4}{*}{0.0001} & 20 & $0.92 \mathrm{E}-02$ & - & $0.20 \mathrm{E}-01$ & - & $0.82 \mathrm{E}-01$ & - \\
\hline & & 40 & $0.62 \mathrm{E}-03$ & 3.89 & $0.12 \mathrm{E}-02$ & 4.07 & $0.51 \mathrm{E}-02$ & 4.00 \\
\hline & & 80 & $0.29 \mathrm{E}-04$ & 4.44 & $0.68 \mathrm{E}-04$ & 4.11 & $0.49 \mathrm{E}-03$ & 3.38 \\
\hline & & 160 & $0.15 \mathrm{E}-05$ & 4.24 & $0.41 \mathrm{E}-05$ & 4.07 & $0.33 \mathrm{E}-04$ & 3.88 \\
\hline \multirow{4}{*}{4} & \multirow{4}{*}{0.0001} & 20 & $0.68 \mathrm{E}-03$ & - & $0.12 \mathrm{E}-02$ & - & $0.49 \mathrm{E}-02$ & - \\
\hline & & 40 & $0.27 \mathrm{E}-04$ & 4.69 & $0.71 \mathrm{E}-04$ & 4.07 & $0.44 \mathrm{E}-03$ & 3.47 \\
\hline & & 80 & $0.61 \mathrm{E}-06$ & 5.47 & $0.18 \mathrm{E}-05$ & 5.30 & $0.12 \mathrm{E}-04$ & 5.20 \\
\hline & & 160 & $0.18 \mathrm{E}-07$ & 5.11 & $0.58 \mathrm{E}-07$ & 4.99 & $0.39 \mathrm{E}-06$ & 4.95 \\
\hline \multirow{4}{*}{5} & \multirow{4}{*}{0.0001} & 20 & $0.10 \mathrm{E}-03$ & - & $0.32 \mathrm{E}-03$ & - & $0.22 \mathrm{E}-02$ & - \\
\hline & & 40 & $0.98 \mathrm{E}-06$ & 6.72 & $0.23 \mathrm{E}-05$ & 7.11 & $0.11 \mathrm{E}-04$ & 7.59 \\
\hline & & 80 & $0.17 \mathrm{E}-07$ & 5.83 & $0.66 \mathrm{E}-07$ & 5.11 & $0.50 \mathrm{E}-06$ & 4.49 \\
\hline & & 160 & $0.26 \mathrm{E}-09$ & 6.06 & $0.10 \mathrm{E}-08$ & 5.98 & $0.93 \mathrm{E}-08$ & 5.74 \\
\hline
\end{tabular}

TABLE 3.4. Errors and numerical orders of accuracy for Example 3.3.4 when using $P^{k}$ polynomials and Runge-Kutta third order time discretization on a uniform mesh of $N$ cells. Final time $T=$ 0.1 .

\begin{tabular}{|c|c|c|c|c|c|c|c|c|}
\hline$k$ & $C F L$ & $N$ & $L^{1}$ error & order & $L^{2}$ error & order & $L^{\infty}$ error & order \\
\hline \multirow{4}{*}{2} & \multirow{4}{*}{0.01} & 20 & $0.59 \mathrm{E}-03$ & - & $0.23 \mathrm{E}-02$ & - & $0.15 \mathrm{E}-01$ & - \\
\hline & & 40 & $0.97 \mathrm{E}-04$ & 2.60 & $0.40 \mathrm{E}-03$ & 2.56 & $0.45 \mathrm{E}-02$ & 1.70 \\
\hline & & 80 & $0.11 \mathrm{E}-04$ & 3.12 & $0.50 \mathrm{E}-04$ & 2.98 & $0.50 \mathrm{E}-03$ & 3.17 \\
\hline & & 160 & $0.19 \mathrm{E}-05$ & 2.57 & $0.80 \mathrm{E}-05$ & 2.65 & $0.66 \mathrm{E}-04$ & 2.91 \\
\hline \multirow{4}{*}{3} & \multirow{4}{*}{0.01} & $\overline{20}$ & $0.22 \mathrm{E}-03$ & - & $0.81 \mathrm{E}-03$ & - & $0.47 \mathrm{E}-02$ & - \\
\hline & & 40 & $0.16 \mathrm{E}-04$ & 3.82 & $0.67 \mathrm{E}-04$ & 3.61 & $0.47 \mathrm{E}-03$ & 3.31 \\
\hline & & 80 & $0.20 \mathrm{E}-05$ & 3.01 & $0.78 \mathrm{E}-05$ & 3.09 & $0.74 \mathrm{E}-04$ & 2.66 \\
\hline & & 160 & $0.21 \mathrm{E}-06$ & 3.23 & $0.95 \mathrm{E}-06$ & 3.04 & $0.13 \mathrm{E}-04$ & 2.56 \\
\hline \multirow{4}{*}{4} & \multirow{4}{*}{0.01} & 20 & $0.87 \mathrm{E}-04$ & - & $0.34 \mathrm{E}-03$ & - & $0.21 \mathrm{E}-02$ & - \\
\hline & & 40 & $0.16 \mathrm{E}-04$ & 2.46 & $0.67 \mathrm{E}-04$ & 2.36 & $0.47 \mathrm{E}-03$ & 2.13 \\
\hline & & 80 & $0.42 \mathrm{E}-06$ & 5.21 & $0.16 \mathrm{E}-05$ & 5.38 & $0.13 \mathrm{E}-04$ & 5.20 \\
\hline & & 160 & $0.18 \mathrm{E}-07$ & 4.55 & $0.74 \mathrm{E}-07$ & 4.43 & $0.72 \mathrm{E}-06$ & 4.15 \\
\hline
\end{tabular}

Example 3.3.4. To test the accuracy of our scheme for nonlinear problems with small coefficients for the third derivative term, we solve the soliton solution of the generalized $\mathrm{KdV}$ equation [27.

$$
u_{t}+u_{x}+\left(\frac{u^{4}}{4}\right)_{x}+\varepsilon u_{x x x}=0
$$


on the domain $[-2,3]$. Here we take $\varepsilon=0.2058 \times 10^{-4}$. The initial condition is

$$
u(x, 0)=A \operatorname{sech}^{\frac{2}{3}}\left(K\left(x-x_{0}\right)\right)
$$

with $A=0.2275, x_{0}=0.5$ and $K=3\left(\frac{A^{3}}{40 \varepsilon}\right)^{\frac{1}{2}}$. The exact solution is

$$
u(x, t)=A \operatorname{sech}^{\frac{2}{3}}\left(K\left(x-x_{0}\right)-\omega t\right)
$$

where $\omega=K\left(1+\frac{A^{3}}{10}\right)$. The boundary conditions are given by

$$
u(-2, t)=g_{1}(t), \quad u_{x}(3, t)=g_{2}(t), \quad u_{x x}(3, t)=g_{3}(t)
$$

where $g_{i}(t)$ corresponds to the data from the exact solution, and we use the upwind flux for the first order convection term and (3.3) as our flux choice. Table 3.4 contains the numerical error and order of accuracy for $2 \leq k \leq 4$. The order of accuracy seems to be less clean for this example, however, at least $k$-th order of accuracy is achieved for the $L^{2}$ norm.

\section{The Convection-Diffusion EQUATion}

Now let us consider the one dimensional convection-diffusion equation

$$
u_{t}+f(u)_{x}-\left(a(u) u_{x}\right)_{x}=0
$$

where $a(u) \geq 0$ is a non-negative function of $u$. We denote $b(u)=\int a(u) d u$ and rewrite the equation into

$$
u_{t}+f(u)_{x}-(b(u))_{x x}=0 .
$$

We propose our DG scheme as follows: find $u_{h} \in V_{h}^{k}$, such that

$$
\begin{aligned}
& \int_{I_{j}}\left(u_{h}\right)_{t} v_{h} d x-\int_{I_{j}} f\left(u_{h}\right)\left(v_{h}\right)_{x} d x-\int_{I_{j}} b\left(u_{h}\right)\left(v_{h}\right)_{x x} d x \\
& +\left(\hat{f}\left(u_{h}^{-}, u_{h}^{+}\right) v_{h}^{-}\right)_{j+\frac{1}{2}}-\left(\widehat{f}\left(u_{h}^{-}, u_{h}^{+}\right) v_{h}^{+}\right)_{j-\frac{1}{2}}-\left(\widetilde{b\left(u_{h}\right)_{x}} v_{h}^{-}\right)_{j+\frac{1}{2}} \\
& +\left(\widetilde{b\left(u_{h}\right)_{x}} v_{h}^{+}\right)_{j-\frac{1}{2}}+\left(\widehat{b\left(u_{h}\right)}\left(v_{h}\right)_{x}^{-}\right)_{j+\frac{1}{2}}-\left(\widehat{b\left(u_{h}\right)}\left(v_{h}\right)_{x}^{+}\right)_{j-\frac{1}{2}}=0
\end{aligned}
$$

holds true for any $v_{h} \in V_{h}^{k}$ and $j=1, \ldots, N$.

Here $\hat{f}\left(u_{h}^{-}, u_{h}^{+}\right)$is a monotone numerical flux, $\widetilde{b\left(u_{h}\right)_{x}}$ and $\widehat{b\left(u_{h}\right)}$ are numerical fluxes that are chosen to be

$$
\widehat{b\left(u_{h}\right)}=b\left(u_{h}^{+}\right), \quad \widetilde{b\left(u_{h}\right)_{x}}=\frac{\left[b\left(u_{h}\right)\right]}{\left[u_{h}\right]}\left(\left(u_{h}\right)_{x}^{-}+\xi\left[b\left(u_{h}\right)\right]\right)
$$

where $\xi$ is a positive constant that is of the order $\mathcal{O}\left(h^{-1}\right)$. Comparing with the LDG method [13, we can notice the appearance of the additional penalty term $\xi\left[b\left(u_{h}\right)\right]$ in the flux $\widetilde{b\left(u_{h}\right)_{x}}$. For the LDG method, such a penalty term is necessary for the elliptic equation [3] but not for the time dependent convection-diffusion equation (4.1). However, we will see later in the stability analysis (also verified by numerical experiments) that the additional jump term $\xi\left[b\left(u_{h}\right)\right]$ is necessary for the stability of our DG scheme. This seems to be a disadvantage of our DG scheme in comparison with the LDG method, however, such an extra penalty term seems to be necessary for our DG scheme only when even-order diffusive type PDEs are solved. 
4.1. Stability analysis. In this subsection, we will examine the stability property of the scheme we just proposed. Again, only a periodic boundary condition is considered.

Theorem 4.1. If $a(u) \geq \beta>0$ for a constant $\beta$, then the $D G$ scheme (4.3) with the flux choice (4.4) is $L^{2}$ stable when $\xi \geq \frac{C}{h}$ for a suitably large constant $C>0$ :

$$
\left\|u_{h}(t)\right\| \leq\left\|u_{h}(0)\right\| \text {. }
$$

Proof. Let $F(u)=\int f(u) d u$. In (4.3), we let $v_{h}=u_{h}$ and sum over $j$ to obtain

$$
\frac{d}{d t} \frac{1}{2} \int_{I} u_{h}^{2} d x+\int_{I} a\left(u_{h}\right)\left(u_{h}\right)_{x}^{2} d x+\sum_{j=1}^{N} \Theta_{j+\frac{1}{2}}=0
$$

where $\Theta_{j+\frac{1}{2}}=\left(\left[F\left(u_{h}\right)\right]-\hat{f}\left(u_{h}^{-}, u_{h}^{+}\right)\left[u_{h}\right]+2\left[b\left(u_{h}\right)\right]\left(u_{h}\right)_{x}^{-}+\xi\left[b\left(u_{h}\right)\right]^{2}\right)_{j+\frac{1}{2}}$. Following the same argument as that in the proof of Theorem 3.1, we have $\left[F\left(u_{h}\right)\right]-$ $\hat{f}\left(u_{h}^{-}, u_{h}^{+}\right)\left[u_{h}\right] \geq 0$. Thus,

$$
\begin{aligned}
& \frac{d}{d t} \frac{1}{2} \int_{I} u_{h}^{2} d x=-\int_{I} a\left(u_{h}\right)\left(u_{h}\right)_{x}^{2} d x-\sum_{j=1}^{N} \Theta_{j+\frac{1}{2}} \\
& \leq-\int_{I} a\left(u_{h}\right)\left(u_{h}\right)_{x}^{2} d x-\sum_{j=1}^{N} \xi\left[b\left(u_{h}\right)\right]_{j+\frac{1}{2}}^{2}+2 \sum_{j=1}^{N}\left|\left[b\left(u_{h}\right)\right]\left(u_{h}\right)_{x}^{-}\right|_{j+\frac{1}{2}} .
\end{aligned}
$$

From the inverse property, there exists a constant $C$, such that

$$
\left(\left(u_{h}\right)_{x}^{-}\right)_{j+1 / 2}^{2} \leq \frac{C}{h} \int_{I_{j}}\left(u_{h}\right)_{x}^{2} d x
$$

so by Young's inequality, for any $\varepsilon>0$, we have

$$
\begin{aligned}
& \frac{d}{d t} \frac{1}{2} \int_{I} u_{h}^{2} d x \\
& \leq-\int_{I} a\left(u_{h}\right)\left(u_{h}\right)_{x}^{2} d x-\sum_{j=1}^{N} \xi\left[b\left(u_{h}\right)\right]_{j+\frac{1}{2}}^{2}+\sum_{j=1}^{N}\left(\varepsilon^{2}\left[b\left(u_{h}\right)\right]^{2}+\frac{\left(\left(u_{h}\right)_{x}^{-}\right)^{2}}{\varepsilon^{2}}\right)_{j+\frac{1}{2}} \\
& \leq\left(\frac{C}{h \varepsilon^{2}}-\beta\right) \int_{I}\left(u_{h}\right)_{x}^{2} d x-\sum_{j=1}^{N}\left(\xi-\varepsilon^{2}\right)\left[b\left(u_{h}\right)\right]_{j+\frac{1}{2}}^{2} .
\end{aligned}
$$

By taking $\varepsilon=\sqrt{\frac{C}{h \beta}}$ and $\xi \geq 2 \varepsilon^{2}=\frac{2 C}{h \beta}$, (4.8) becomes

$$
\frac{d}{d t} \frac{1}{2} \int_{I} u_{h}^{2} d x \leq-\frac{C}{h \beta} \sum_{j=1}^{N}\left[u_{h}\right]_{j+\frac{1}{2}}^{2} \leq 0,
$$

which finishes the proof.

4.2. Error estimate. In this subsection, we state the error estimate of our scheme for the linear diffusion case $a(u)=1$. The rather technical proof of this result is again left to the Appendix.

Theorem 4.2. Let $u$ be the exact solution of the equation (4.1) with $a(u)=1$, which is sufficiently smooth with bounded derivatives, and assume $f \in C^{3}$. Let $u_{h}$ be the numerical solution of (4.3) with (4.4) as our flux choice with $\xi=\frac{C}{h}$ for the 
same $C$ as that in Theorem 4.1. If we impose periodic boundary condition, and use $V_{h}^{k}$ space with $k \geq 1$, then we have an error estimate as

$$
\left\|u(t)-u_{h}(t)\right\| \leq C h^{k}
$$

where the constant $C$ depends on $k, t,\|u\|_{k+1}$ and the bounds on the derivatives $\left|f^{(m)}\right|, m=1,2,3$, but not on $h$. For $k=1$, we also require the convection term to be linear $f(u)=c u$.

Proof. The proof of this theorem is provided in the Appendix; see Section A.4.

Remark. When $k=0$, numerical experiments show that our scheme is not consistent.

4.3. A numerical example. In this subsection, we give a numerical example to demonstrate the performance of our scheme.

\section{Example 4.3.1.}

$$
\left\{\begin{array}{l}
u_{t}-u_{x x}=0 \\
u(x, 0)=\sin (x), \\
u(0, t)=u(2 \pi, t) .
\end{array}\right.
$$

The exact solution is given by

$$
u(x, t)=e^{-t} \sin (x) .
$$

In our computation below, we use the backward Euler time discretization. However, in order for the time error not to dominate, we still use a small time step $\triangle t=C F L h^{k+1}$ for the $V_{h}^{k}$ space. The errors and numerical order of accuracy for $P^{k}$ elements with $0 \leq k \leq 3$ are listed in Table 4.1. We observe that our scheme is not consistent for $P^{0}$ polynomials, while optimal $(k+1)$-th order of accuracy is achieved for $k \geq 1$. We also remark that the additional jump term $\xi\left[u_{h}\right]$ in the flux is necessary for stability. Without this term, when $k=3, N=80$, and the numerical solution blows up.

4.4. Remark on another DG scheme. For (4.1), we can propose another DG scheme that is formulated by one integration by parts instead of two: find $u_{h} \in V_{h}^{k}$, such that

$$
\begin{aligned}
& \int_{I_{j}}\left(u_{h}\right)_{t} v_{h} d x-\int_{I_{j}} f\left(u_{h}\right)\left(v_{h}\right)_{x} d x+\int_{I_{j}} a\left(u_{h}\right)\left(u_{h}\right)_{x}\left(v_{h}\right)_{x} d x \\
& +\left(\hat{f}\left(u_{h}^{-}, u_{h}^{+}\right) v_{h}^{-}\right)_{j+\frac{1}{2}}-\left(\hat{f}\left(u_{h}^{-}, u_{h}^{+}\right) v_{h}^{+}\right)_{j-\frac{1}{2}} \\
& -\left(a\left(\widetilde{\left.u_{h}\right)\left(u_{h}\right.}\right)_{x} v_{h}^{-}\right)_{j+\frac{1}{2}}+\left(a\left(u_{h}\right)\left(u_{h}\right)_{x} v_{h}^{+}\right)_{j-\frac{1}{2}}=0
\end{aligned}
$$

holds for any $v_{h} \in V_{h}^{k}$ and $j=1, \ldots, N$. We take the numerical flux to be $a\left(\widetilde{\left.u_{h}\right)\left(u_{h}\right.}\right)_{x}=a\left(u_{h}^{-}\right)\left(\left(u_{h}\right)_{x}^{-}+\xi\left[u_{h}\right]\right)$ with the same choice of $\xi$ as before. Then $L^{2}$ stability can be proven without the positivity restriction $a(u) \geq \beta>0$ as in Theorem 4.2 , rather $a(u) \geq 0$ suffices. Numerical experiments on the heat equation, however, indicate that this scheme is only $k$-th order accurate when $P^{k}$ polynomials are used. 
TABLE 4.1. Errors and numerical orders of accuracy for Example 4.3.1 when using $P^{k}$ polynomials and backward Euler time discretization on a uniform mesh of $N$ cells. Final time $T=1 . \xi=$ $10 / h$.

\begin{tabular}{|c|c|c|c|c|c|c|c|c|}
\hline$k$ & $C F L$ & $N$ & $L^{1}$ error & order & $L^{2}$ error & order & $L^{\infty}$ error & order \\
\hline \multirow{3}{*}{0} & \multirow{3}{*}{1} & 10 & $0.21 \mathrm{E}+00$ & - & $0.24 \mathrm{E}+00$ & - & $0.34 \mathrm{E}+00$ & - \\
\hline & & 20 & $0.23 \mathrm{E}+00$ & -0.09 & $0.25 \mathrm{E}+00$ & -0.09 & $0.36 \mathrm{E}+00$ & -0.09 \\
\hline & & 40 & $0.23 \mathrm{E}+00$ & $\begin{array}{l}-0.03 \\
\end{array}$ & $0.26 \mathrm{E}+00$ & $\begin{array}{l}-0.03 \\
\end{array}$ & $0.37 \mathrm{E}+00$ & -0.03 \\
\hline \multirow{4}{*}{1} & \multirow{4}{*}{1} & 10 & $0.32 \mathrm{E}-01$ & - & $0.36 \mathrm{E}-01$ & - & $0.63 \mathrm{E}-01$ & - \\
\hline & & 20 & $0.97 \mathrm{E}-02$ & 1.70 & $0.11 \mathrm{E}-01$ & 1.71 & $0.19 \mathrm{E}-01$ & 1.74 \\
\hline & & 40 & $0.25 \mathrm{E}-02$ & 1.95 & $0.28 \mathrm{E}-02$ & 1.95 & $0.48 \mathrm{E}-02$ & 1.97 \\
\hline & & 80 & $0.64 \mathrm{E}-03$ & 1.98 & $0.71 \mathrm{E}-03$ & 1.98 & $0.12 \mathrm{E}-02$ & 1.99 \\
\hline \multirow{4}{*}{2} & \multirow{4}{*}{1} & 10 & $0.26 \mathrm{E}-01$ & - & $0.29 \mathrm{E}-01$ & - & $0.41 \mathrm{E}-01$ & - \\
\hline & & 20 & $0.36 \mathrm{E}-02$ & 2.88 & $0.40 \mathrm{E}-02$ & 2.88 & $0.56 \mathrm{E}-02$ & 2.87 \\
\hline & & 40 & $0.45 \mathrm{E}-03$ & 2.98 & $0.50 \mathrm{E}-03$ & 2.98 & $0.71 \mathrm{E}-03$ & 2.98 \\
\hline & & 80 & $0.57 \mathrm{E}-04$ & 3.00 & $0.63 \mathrm{E}-04$ & 3.00 & $0.89 \mathrm{E}-04$ & 3.00 \\
\hline \multirow{4}{*}{3} & \multirow{4}{*}{1} & 10 & $0.17 \mathrm{E}-01$ & - & $0.18 \mathrm{E}-01$ & - & $0.26 \mathrm{E}-01$ & - \\
\hline & & 20 & $0.11 \mathrm{E}-02$ & 3.86 & $0.13 \mathrm{E}-02$ & 3.86 & $0.18 \mathrm{E}-02$ & 3.86 \\
\hline & & 40 & $0.71 \mathrm{E}-04$ & 3.99 & $0.79 \mathrm{E}-04$ & 3.99 & $0.11 \mathrm{E}-03$ & 3.99 \\
\hline & & 80 & $0.45 \mathrm{E}-05$ & 4.00 & $0.49 \mathrm{E}-05$ & 4.00 & $0.70 \mathrm{E}-05$ & 4.00 \\
\hline
\end{tabular}

\section{Equations With Higher ORDER DERIVATIVES}

In this section, we will generalize our scheme to deal with equations with higher order derivatives.

5.1. A fifth order nonlinear equation. Our scheme can be applied to the following equation:

$$
u_{t}+f(u)_{x}+\sigma u_{x x x x x}=0 .
$$

We propose a scheme as follows: find $u_{h} \in V_{h}^{k}$, such that

$$
\begin{aligned}
& \int_{I_{j}}\left(u_{h}\right)_{t} v_{h} d x-\int_{I_{j}} f\left(u_{h}\right)\left(v_{h}\right)_{x} d x+\left(\hat{f}\left(u_{h}^{-}, u_{h}^{+}\right) v_{h}^{-}\right)_{j+\frac{1}{2}}-\left(\hat{f}\left(u_{h}^{-}, u_{h}^{+}\right) v_{h}^{+}\right)_{j-\frac{1}{2}} \\
& -\sigma \int_{I_{j}} u_{h}\left(v_{h}\right)_{x x x x x} d x+\sigma\left(\hat{u_{h}}\left(v_{h}\right)_{x x x x}^{-}\right)_{j+\frac{1}{2}}-\sigma\left(\hat{u_{h}}\left(v_{h}\right)_{x x x x}^{+}\right)_{j-\frac{1}{2}} \\
& -\sigma\left(\left(\tilde{u_{h}}\right)_{x}\left(v_{h}\right)_{x x x}^{-}\right)_{j+\frac{1}{2}}+\sigma\left(\left(\tilde{u}_{h}\right)_{x}\left(v_{h}\right)_{x x x}^{+}\right)_{j-\frac{1}{2}}+\sigma\left(\left(\bar{u}_{h}\right)_{x x}\left(v_{h}\right)_{x x}^{-}\right)_{j+\frac{1}{2}} \\
& -\sigma\left(\left(\left(\bar{u}_{h}\right)_{x x}\left(v_{h}\right)_{x x}^{+}\right)_{j-\frac{1}{2}}-\sigma\left(\left(\tilde{u}_{h}\right)_{x x x}\left(v_{h}\right)_{x}^{-}\right)_{j+\frac{1}{2}}+\sigma\left(\left(\check{u}_{h}\right)_{x x x}\left(v_{h}\right)_{x}^{+}\right)_{j-\frac{1}{2}}\right. \\
& +\sigma\left(\left(\dot{u}_{h}\right)_{x x x x} v_{h}^{-}\right)_{j+\frac{1}{2}}-\sigma\left(\left(\dot{u}_{h}\right)_{x x x x} v_{h}^{+}\right)_{j-\frac{1}{2}}=0
\end{aligned}
$$

holds for any $v_{h} \in V_{h}^{k}$ and $j=1, \ldots, N$. In (5.2), $\hat{f}\left(u_{h}^{-}, u_{h}^{+}\right)$is a monotone numerical flux. The pair of fluxes $\hat{u_{h}}$ and $\left(\dot{u_{h}}\right)_{x x x x}$ should be taken from the opposite directions of each other; likewise for the pair $\left(\tilde{u_{h}}\right)_{x}$ and $\left(\tilde{u_{h}}\right)_{x x x}$. If $\sigma>0$, then $\left(\overline{u_{h}}\right)_{x x}=\left(u_{h}\right)_{x x}^{-}$; otherwise, $\left(\overline{u_{h}}\right)_{x x}=\left(u_{h}\right)_{x x}^{+}$. Therefore, for each fixed $\sigma$, there are four combinations of flux choices available.

For simplicity of discussion, we will only consider the case $\sigma>0$ from now on. 
5.1.1. Stability analysis. In this subsection, we will examine the stability property of the scheme (5.2). As before, we assume periodic boundary condition.

Theorem 5.1. Our numerical scheme (5.2) is $L^{2}$ stable for all four flux choices, i.e.

$$
\left\|u_{h}(t)\right\| \leq\left\|u_{h}(0)\right\| .
$$

Proof. The proof is almost the same as the proof of Theorem 3.1. Let $F(u)=$ $\int f(u) d u$. In (5.2), we take $v_{h}=u_{h}$ and sum over $j$ to obtain

$$
\frac{d}{d t} \frac{1}{2} \int_{I} u_{h}^{2} d x+\sum_{j=1}^{N} \Theta_{j-\frac{1}{2}}=0
$$

where $\Theta_{j-\frac{1}{2}}=\left(\left[F\left(u_{h}\right)\right]-\hat{f}\left(u_{h}^{-}, u_{h}^{+}\right)\left[u_{h}\right]+\frac{\sigma}{2}\left[\left(u_{h}\right)_{x x}\right]^{2}\right)_{j-\frac{1}{2}}$. This holds for all four flux choices. Now, by the property of a monotone flux $\hat{f}$, we can show that $\left[F\left(u_{h}\right)\right]-$ $\hat{f}\left(u_{h}^{-}, u_{h}^{+}\right)\left[u_{h}\right] \geq 0$, so that $\Theta_{j-\frac{1}{2}} \geq 0$, which finishes the proof.

5.1.2. Error estimates. In this subsection, we state the error estimates of our scheme. The proof of these results is left to the Appendix.

Theorem 5.2. Let $u$ be the exact solution of the equation (5.1), which is sufficiently smooth with bounded derivatives, and assume $f \in C^{3}$. Let $u_{h}$ be the numerical solution of (5.2). If we impose periodic boundary condition, and use $V_{h}^{k}$ space with $k \geq 5$, then we have an error estimate as

$$
\left\|u_{h}(t)-u(t)\right\| \leq C h^{k}
$$

where the constant $C$ depends on $k, t,\|u\|_{k+1}$ and the bounds on the derivatives $\left|f^{(m)}\right|, m=1,2,3$, but not on $h$.

Proof. The proof of this theorem is provided in the Appendix; see Section A.5.

Remark. If $f(u)=0$, then the result of Theorem 5.2 can be improved to the optimal $O\left(h^{k+1}\right)$ error estimate.

Theorem 5.2 only deals with the case of $k \geq 5$. If $k=4$, the following theorem gives a more sub-optimal error estimate.

Theorem 5.3. Under the same condition of Theorem 5.2, if $k=4$, then we have an error estimate as

$$
\left\|u_{h}(t)-u(t)\right\| \leq C h^{\frac{5}{2}}
$$

where the constant $C$ depends on $t,\|u\|_{k+1}$ and the bounds on the derivatives $\left|f^{(m)}\right|$, $m=1,2,3$, but not on $h$.

Proof. The proof of this theorem is provided in the Appendix; see Section A.6

Remark. When $k<4$, numerical experiments show that our scheme is not consistent. 
5.1.3. A numerical example. In this subsection, we give a numerical example.

Example 5.1.1. Consider the fifth order linear equation

$$
\left\{\begin{array}{l}
u_{t}+u_{x}+u_{x x x x x}=0, \\
u(x, 0)=\sin (x), \\
u(0, t)=u(2 \pi, t) .
\end{array}\right.
$$

The exact solution is given by

$$
u(x, t)=\sin (x-2 t) .
$$

We test the DG scheme with the following flux choice: $\hat{u}_{h}=u_{h}^{-},\left(\tilde{u_{h}}\right)_{x}=\left(u_{h}\right)_{x}^{-}$, $\left(\overline{u_{h}}\right)_{x x}=\left(u_{h}\right)_{x x}^{-},\left(\check{u}_{h}\right)_{x x x}=\left(u_{h}\right)_{x x x}^{+}$and $\left(\dot{u_{h}}\right)_{x x x x}=\left(u_{h}\right)_{x x x x}^{+}$. For the first order convection term, we will just use the upwind flux, i.e. $\hat{f}\left(u_{h}^{-}, u_{h}^{+}\right)=u_{h}^{-}$. We use the backward Euler time discretization with a small time step in order for the time error not to dominate. The errors and numerical order of accuracy for $P^{k}$ elements with $3 \leq k \leq 5$ are listed in Table 5.1 . We observe that our scheme is not consistent for $P^{3}$ polynomials, while optimal $(k+1)$-th order of accuracy is achieved for $k \geq 4$.

TABLE 5.1. Errors and numerical orders of accuracy for Example 5.1.1 when using $P^{k}$ polynomials and backward Euler time discretization on a uniform mesh of $N$ cells. Final time $T=1 . \triangle t=$ $C F L h^{k+1}$.

\begin{tabular}{|c|c|c|cc|cc|cc|}
\hline$k$ & $C F L$ & $N$ & $L^{1}$ error & order & $L^{2}$ error & order & $L^{\infty}$ error & order \\
\hline \multirow{3}{*}{3} & \multirow{3}{*}{1} & 10 & $0.54 \mathrm{E}+00$ & - & $0.61 \mathrm{E}+00$ & - & $0.10 \mathrm{E}+01$ & - \\
\cline { 3 - 9 } & & 20 & $0.56 \mathrm{E}+00$ & -0.06 & $0.63 \mathrm{E}+00$ & -0.05 & $0.10 \mathrm{E}+01$ & 0.00 \\
\hline & & 40 & $0.59 \mathrm{E}+00$ & -0.06 & $0.65 \mathrm{E}+00$ & -0.05 & $0.98 \mathrm{E}+00$ & 0.02 \\
\hline \multirow{4}{*}{4} & \multirow{3}{*}{1} & 10 & $0.11 \mathrm{E}+00$ & - & $0.12 \mathrm{E}+00$ & - & $0.17 \mathrm{E}+00$ & - \\
\cline { 3 - 9 } & & 20 & $0.39 \mathrm{E}-02$ & 4.83 & $0.43 \mathrm{E}-02$ & 4.83 & $0.61 \mathrm{E}-02$ & 4.83 \\
\cline { 3 - 9 } & & 40 & $0.12 \mathrm{E}-03$ & 4.99 & $0.14 \mathrm{E}-03$ & 4.99 & $0.19 \mathrm{E}-03$ & 4.99 \\
\hline & & 80 & $0.36 \mathrm{E}-05$ & 5.08 & $0.40 \mathrm{E}-05$ & 5.08 & $0.57 \mathrm{E}-05$ & 5.08 \\
\hline \multirow{3}{*}{5} & \multirow{3}{*}{1} & 10 & $0.73 \mathrm{E}-01$ & - & $0.81 \mathrm{E}-01$ & - & $0.11 \mathrm{E}+00$ & - \\
\cline { 3 - 9 } & & 20 & $0.12 \mathrm{E}-02$ & 5.89 & $0.14 \mathrm{E}-02$ & 5.89 & $0.19 \mathrm{E}-02$ & 5.89 \\
\cline { 3 - 9 } & & 40 & $0.19 \mathrm{E}-04$ & 6.00 & $0.21 \mathrm{E}-04$ & 6.00 & $0.30 \mathrm{E}-04$ & 6.00 \\
\hline
\end{tabular}

5.2. A time dependent biharmonic equation. Now let us consider a time dependent biharmonic equation given by

$$
u_{t}+f(u)_{x}+\sigma u_{x x x x}=0
$$

where $\sigma>0$ is a constant.

We propose a DG scheme as follows: find $u_{h} \in V_{h}^{k}$, such that

$$
\begin{aligned}
& \int_{I_{j}}\left(u_{h}\right)_{t} v_{h} d x-\int_{I_{j}} f\left(u_{h}\right)\left(v_{h}\right)_{x} d x+\sigma \int_{I_{j}} u_{h}\left(v_{h}\right)_{x x x x} d x \\
& +\left(\hat{f}\left(u_{h}^{-}, u_{h}^{+}\right) v_{h}^{-}\right)_{j+\frac{1}{2}}-\left(\hat{f}\left(u_{h}^{-}, u_{h}^{+}\right) v_{h}^{+}\right)_{j-\frac{1}{2}}-\sigma\left(\tilde{u_{h}}\left(v_{h}\right)_{x x x}^{-}\right)_{j+\frac{1}{2}} \\
& +\sigma\left(\tilde{u_{h}}\left(v_{h}\right)_{x x x}^{+}\right)_{j-\frac{1}{2}}+\sigma\left(\left(\tilde{u_{h}}\right)_{x}\left(v_{h}\right)_{x x}^{-}\right)_{j+\frac{1}{2}}-\sigma\left(\left(\tilde{u_{h}}\right)_{x}\left(v_{h}\right)_{x x}^{+}\right)_{j-\frac{1}{2}} \\
& -\sigma\left(\left(\overline{u_{h}}\right)_{x x}\left(v_{h}\right)_{x}^{-}\right)_{j+\frac{1}{2}}+\sigma\left(\left(\overline{u_{h}}\right)_{x x}\left(v_{h}\right)_{x}^{+}\right)_{j-\frac{1}{2}}+\sigma\left(\left(\hat{u_{h}}\right)_{x x x} v_{h}^{-}\right)_{j+\frac{1}{2}} \\
& -\sigma\left(\left(\hat{u_{h}}\right)_{x x x} v_{h}^{+}\right)_{j-\frac{1}{2}}=0
\end{aligned}
$$

holds for any $v_{h} \in V_{h}^{k}$ and $j=1, \ldots, N$. 
Here $\hat{f}\left(u_{h}^{-}, u_{h}^{+}\right)$is a monotone numerical flux. $\tilde{u_{h}},\left(\tilde{u_{h}}\right)_{x},\left(\overline{u_{h}}\right)_{x x}$ and $\left(\hat{u_{h}}\right)_{x x x}$ are numerical fluxes that can be chosen as

$$
\tilde{u_{h}}=u_{h}^{+}, \quad\left(\tilde{u_{h}}\right)_{x}=\left(u_{h}\right)_{x}^{-}, \quad\left(\overline{u_{h}}\right)_{x x}=\left(u_{h}\right)_{x x}^{+}, \quad\left(\hat{u_{h}}\right)_{x x x}=\left(u_{h}\right)_{x x x}^{-}-\xi\left[u_{h}\right]
$$

where $\xi$ is a positive constant that is of the order $\mathcal{O}\left(h^{-3}\right)$. Comparing with the LDG method 28, we again notice the appearance of the additional penalty term $\xi\left[u_{h}\right]$ in the flux $\left(\hat{u_{h}}\right)_{x x x}$. For the LDG method, such a penalty term is necessary for the steady state biharmonic equation but not for the time dependent biharmonic equation [28. However, we will see later in the stability analysis (also verified by numerical experiments) that the additional jump term $\xi\left[u_{h}\right]$ is necessary for the stability of our DG scheme.

5.2.1. Stability analysis. Here, we follow the lines of Section 4.1 and examine the stability property of the scheme we just proposed. For simplicity, let us impose the periodic boundary condition.

Theorem 5.4. The DG scheme (5.9) with the flux choice (5.10) is $L^{2}$ stable when $\xi \geq \frac{C}{h^{3}}$ for a suitably chosen constant $C>0$ :

$$
\left\|u_{h}(t)\right\| \leq\left\|u_{h}(0)\right\| .
$$

Proof. Let $F(u)=\int f(u) d u$. In (5.9), we let $v_{h}=u_{h}$ and sum over $j$ to obtain

$$
\frac{d}{d t} \frac{1}{2} \int_{I} u_{h}^{2} d x+\int_{I}\left(u_{h}\right)_{x x}^{2} d x+\sum_{j=1}^{N} \Theta_{j-\frac{1}{2}}=0
$$

where $\Theta_{j-\frac{1}{2}}=\left(\left[F\left(u_{h}\right)\right]-\hat{f}\left(u_{h}^{-}, u_{h}^{+}\right)\left[u_{h}\right]-2 \sigma\left[u_{h}\right]\left(u_{h}\right)_{x x x}^{-}+\xi \sigma\left[u_{h}\right]^{2}\right)_{j-\frac{1}{2}}$. From the same deduction as in the proof of Theorem 3.1, we have $\left[F\left(u_{h}\right)\right]-\hat{f}\left(u_{h}^{-}, u_{h}^{+}\right)\left[u_{h}\right] \geq 0$. Thus,

$$
\begin{aligned}
\frac{d}{d t} \frac{1}{2} \int_{I} u_{h}^{2} d x & =-\int_{I}\left(u_{h}\right)_{x x}^{2} d x-\sum_{j=1}^{N} \Theta_{j-\frac{1}{2}} \\
& \leq-\int_{I}\left(u_{h}\right)_{x x}^{2} d x-\sigma \sum_{j=1}^{N} \xi\left[u_{h}\right]_{j+\frac{1}{2}}^{2}+2 \sigma \sum_{j=1}^{N}\left|\left[u_{h}\right]\left(u_{h}\right)_{x x x}^{-}\right|_{j+\frac{1}{2}} .
\end{aligned}
$$

From the inverse property, there exists a constant $C$, such that

$$
\left(\left(u_{h}\right)_{x x x}^{-}\right)_{j+1 / 2}^{2} \leq \frac{C}{h} \int_{I_{j}}\left(u_{h}\right)_{x x x}^{2} d x \leq \frac{C}{h^{3}} \int_{I_{j}}\left(u_{h}\right)_{x x}^{2} d x .
$$

So by Young's inequality, for any $\varepsilon>0$, we have

$$
\begin{aligned}
& \frac{d}{d t} \frac{1}{2} \int_{I} u_{h}^{2} d x \\
& \leq-\int_{I}\left(u_{h}\right)_{x x}^{2} d x-\sigma \sum_{j=1}^{N} \xi\left[u_{h}\right]_{j+\frac{1}{2}}^{2}+\sigma \sum_{j=1}^{N}\left(\varepsilon^{2}\left[u_{h}\right]^{2}+\frac{\left(\left(u_{h}\right)_{x x x}^{-}\right)^{2}}{\varepsilon^{2}}\right)_{j+\frac{1}{2}} \\
& \leq\left(\frac{\sigma C}{h^{3} \varepsilon^{2}}-1\right) \int_{I}\left(u_{h}\right)_{x x}^{2} d x-\sigma \sum_{j=1}^{N}\left(\xi-\varepsilon^{2}\right)\left[u_{h}\right]_{j+\frac{1}{2}}^{2} .
\end{aligned}
$$


By taking $\varepsilon=\sqrt{\frac{\sigma C}{h^{3}}}$ and $\xi \geq 2 \varepsilon^{2}=\frac{2 \sigma C}{h^{3}}$, (5.14) becomes

$$
\frac{d}{d t} \frac{1}{2} \int_{I} u_{h}^{2} d x \leq-\sigma \frac{C}{h^{3}} \sum_{j=1}^{N}\left[u_{h}\right]_{j+\frac{1}{2}}^{2} \leq 0
$$

which finishes the proof.

5.2.2. Error estimate. In this subsection, we state the error estimate of our scheme and leave the proof to the Appendix.

Theorem 5.5. Let $u$ be the exact solution of the equation (5.8), which is sufficiently smooth with bounded derivatives, and assume $f \in C^{3}$. Let $u_{h}$ be the numerical solution of (5.9) with (5.10) as our flux choice with $\xi=\frac{C}{h^{3}}$ for the same $C$ as in Theorem 5.4. If we impose the periodic boundary condition, and use $V_{h}^{k}$ space with $k \geq 3$, then we have an error estimate as

$$
\left\|u_{h}(t)-u(t)\right\| \leq C h^{k-1}
$$

where the constant $C$ depends on $k, t,\|u\|_{k+1}$ and the bounds on the derivatives $\left|f^{(m)}\right|, m=1,2,3$, but not on $h$.

Proof. The proof of this theorem is provided in the Appendix; see Section A.7.

5.2.3. A numerical example. In this subsection, we give a numerical example.

Example 5.2.1. Consider the linear biharmonic equation

$$
\left\{\begin{array}{l}
u_{t}+u_{x x x x}=0 \\
u(x, 0)=\sin (x) \\
u(0, t)=u(2 \pi, t)
\end{array}\right.
$$

The exact solution is given by

$$
u(x, t)=e^{-t} \sin (x) .
$$

In our computation below, we will use the backward Euler time discretization. For the $V_{h}^{k}$ space, we require that time step $\triangle t=C F L h^{k+1}$. Our scheme turns out to be inconsistent for $P^{k}$ polynomials with $k<2$. For the $P^{2}$ case, although we cannot prove its convergence, numerical results from Table 5.2 show second order accuracy, which is one order lower than optimal. For the $k>2$ case, our scheme achieves the optimal $(k+1)$-th order accuracy.

5.3. The fifth order fully nonlinear $K(n, n, n)$ equation. The fifth-order fully nonlinear $K(n, n, n)$ equation is given by

$$
u_{t}+\left(u^{n}\right)_{x}+\left(u^{n}\right)_{x x x}+\left(u^{n}\right)_{x x x x x}=0 .
$$

For the following discussions, we need to use the $L^{n+1}$ norm, defined as $\|u\|_{L^{n+1}}^{n+1}=$ $\int_{I}|u(x)|^{n+1} d x$, of a function $u$ in the space $L^{n+1}=\left\{f(x):\|f\|_{L^{n+1}}<\infty\right\}$. An LDG method for this equation is given in $\left[22\right.$, which can be proved to be $L^{n+1}$ stable when $n$ is odd. Here, we introduce a DG scheme with the same $L^{n+1}$ stability 
TABLE 5.2. Errors and numerical orders of accuracy for Example 5.2.1 when using $P^{k}$ polynomials and backward Euler time discretization on a uniform mesh of $N$ cells. Final time $T=1$. $\xi=$ $10 / h^{3}$.

\begin{tabular}{|c|c|c|cc|cc|cc|}
\hline$k$ & $C F L$ & $N$ & $L^{1}$ error & order & $L^{2}$ error & order & $L^{\infty}$ error & order \\
\hline \multirow{4}{*}{2} & \multirow{4}{*}{1} & 10 & $0.71 \mathrm{E}-02$ & - & $0.79 \mathrm{E}-02$ & - & $0.12 \mathrm{E}-01$ & - \\
\cline { 3 - 9 } & & 20 & $0.18 \mathrm{E}-02$ & 1.98 & $0.20 \mathrm{E}-02$ & 1.98 & $0.30 \mathrm{E}-02$ & 2.04 \\
\cline { 3 - 9 } & 40 & $0.89 \mathrm{E}-03$ & 1.00 & $0.99 \mathrm{E}-03$ & 1.00 & $0.14 \mathrm{E}-02$ & 1.09 \\
\hline & & 80 & $0.28 \mathrm{E}-03$ & 1.67 & $0.31 \mathrm{E}-03$ & 1.67 & $0.44 \mathrm{E}-03$ & 1.68 \\
\hline & & 160 & $0.77 \mathrm{E}-04$ & 1.86 & $0.86 \mathrm{E}-04$ & 1.86 & $0.12 \mathrm{E}-03$ & 1.86 \\
\hline \multirow{4}{*}{3} & \multirow{4}{*}{1} & 10 & $0.18 \mathrm{E}-01$ & - & $0.20 \mathrm{E}-01$ & - & $0.30 \mathrm{E}-01$ & - \\
\cline { 3 - 9 } & & 20 & $0.13 \mathrm{E}-02$ & 3.87 & $0.14 \mathrm{E}-02$ & 3.87 & $0.21 \mathrm{E}-02$ & 3.84 \\
\cline { 3 - 9 } & & 40 & $0.79 \mathrm{E}-04$ & 3.99 & $0.88 \mathrm{E}-04$ & 3.99 & $0.13 \mathrm{E}-03$ & 3.99 \\
\hline & & 80 & $0.49 \mathrm{E}-05$ & 4.00 & $0.55 \mathrm{E}-05$ & 4.00 & $0.82 \mathrm{E}-05$ & 4.00 \\
\hline \multirow{4}{*}{4} & \multirow{3}{*}{1} & 10 & $0.11 \mathrm{E}-01$ & - & $0.12 \mathrm{E}-01$ & - & $0.17 \mathrm{E}-01$ & - \\
\cline { 3 - 8 } & & 20 & $0.36 \mathrm{E}-03$ & 4.92 & $0.40 \mathrm{E}-03$ & 4.92 & $0.56 \mathrm{E}-03$ & 4.92 \\
\hline & & 40 & $0.11 \mathrm{E}-04$ & 5.00 & $0.12 \mathrm{E}-04$ & 5.00 & $0.18 \mathrm{E}-04$ & 5.00 \\
\hline
\end{tabular}

for odd $n$. Our scheme is formulated as to find $u_{h}, w_{h} \in V_{h}^{k}$, such that

$$
\begin{aligned}
& \int_{I_{j}}\left(u_{h}\right)_{t} v_{h} d x-\int_{I_{j}} w_{h}\left(v_{h}\right)_{x} d x-\int_{I_{j}} w_{h}\left(v_{h}\right)_{x x x} d x-\int_{I_{j}} w_{h}\left(v_{h}\right)_{x x x x x} d x \\
& +\left(\bar{w}_{h} v_{h}^{-}\right)_{j+\frac{1}{2}}-\left(\overline{w_{h}} v_{h}^{+}\right)_{j-\frac{1}{2}} \\
& +\left(\left(\tilde{w}_{h}\right)_{x x} v_{h}^{-}\right)_{j+\frac{1}{2}}-\left(\left(\tilde{w}_{h}\right)_{x x} v_{h}^{+}\right)_{j-\frac{1}{2}}-\left(\left(\tilde{w}_{h}\right)_{x}\left(v_{h}\right)_{x}^{-}\right)_{j+\frac{1}{2}} \\
& +\left(\left(\tilde{w}_{h}\right)_{x}\left(v_{h}\right)_{x}^{+}\right)_{j-\frac{1}{2}}+\left(\left(\tilde{w}_{h}\right)\left(v_{h}\right)_{x x}^{-}\right)_{j+\frac{1}{2}}-\left(\left(\tilde{w}_{h}\right)\left(v_{h}\right)_{x x}^{+}\right)_{j-\frac{1}{2}} \\
& +\left(\hat{w}_{h}\left(v_{h}\right)_{x x x x}^{-}\right)_{j+\frac{1}{2}}-\left(\hat{w}_{h}\left(v_{h}\right)_{x x x x}^{+}\right)_{j-\frac{1}{2}}-\left(\left(\hat{w}_{h}\right)_{x}\left(v_{h}\right)_{x x x}^{-}\right)_{j+\frac{1}{2}} \\
& +\left(\left(\hat{w_{h}}\right)_{x}\left(v_{h}\right)_{x x x}^{+}\right)_{j-\frac{1}{2}}+\left(\left(\hat{w}_{h}\right)_{x x}\left(v_{h}\right)_{x x}^{-}\right)_{j+\frac{1}{2}}-\left(\left(\hat{w_{h}}\right)_{x x}\left(v_{h}\right)_{x x}^{+}\right)_{j-\frac{1}{2}} \\
& -\left(\left(\hat{w_{h}}\right)_{x x x}\left(v_{h}\right)_{x}^{-}\right)_{j+\frac{1}{2}}+\left(\left(\hat{w_{h}}\right)_{x x x}\left(v_{h}\right)_{x}^{+}\right)_{j-\frac{1}{2}}+\left(\left(\hat{w_{h}}\right)_{x x x x} v_{h}^{-}\right)_{j+\frac{1}{2}} \\
& -\left(\left(\hat{w_{h}}\right)_{x x x x} v_{h}^{+}\right)_{j-\frac{1}{2}}=0
\end{aligned}
$$

and

$$
\int_{I_{j}} w_{h} g_{h} d x=\int_{I_{j}} u_{h}^{n} g_{h} d x
$$

hold true for any $v_{h}, g_{h} \in V_{h}^{k}$ and $j=1, \ldots, N$. In this scheme, $w_{h}$ is the $L^{2}$ projection of $u_{h}^{n}$ into the finite element space $V_{h}^{k}$. All the "hat" terms are numerical fluxes. The second line of (5.20) contains the numerical fluxes from integration by parts of the term $w_{x}$ and they should take the form $w_{h}^{-}$. The third and fourth line contains flux terms from $w_{x x x}$ and the flux choice is the same as that in Section 3. namely, $\left(\tilde{w}_{h}\right)_{x}=\left(w_{h}\right)_{x}^{+}$and $\tilde{w}_{h},\left(\tilde{w}_{h}\right)_{x x}$ from opposite directions. The fifth to eighth lines contain flux terms induced by $\left(w_{h}\right)_{x x x x x}$. The choices are the same as those in Section 5.1 namely, $\left(\hat{w}_{h}\right)_{x x}=\left(w_{h}\right)_{x x}^{-}$and $\hat{w}_{h},\left(\hat{w}_{h}\right)_{x x x x}$ and $\left(\hat{w}_{h}\right)_{x},\left(\hat{w}_{h}\right)_{x x x}$ from opposite directions of each other. We will see in the following theorem that this flux choice will give us a $L^{n+1}$ stable scheme for odd $n$. 
Theorem 5.6. The following inequality holds

$$
\frac{d}{d t} \int_{I} u_{h}^{n+1} d x \leq 0
$$

In particular, our numerical scheme for (5.19) is $L^{n+1}$ stable for odd $n$ :

$$
\left\|u_{h}(t)\right\|_{L^{n+1}} \leq\left\|u_{h}(0)\right\|_{L^{n+1}} .
$$

Proof. Letting $v_{h}=w_{h}$ in our scheme and summing over $j$, we obtain

$$
\int_{I}\left(u_{h}\right)_{t} w_{h} d x+\sum_{j=1}^{N} \Theta_{j-\frac{1}{2}}=0
$$

where $\Theta_{j-\frac{1}{2}}=\frac{1}{2}\left[w_{h}\right]_{j-\frac{1}{2}}^{2}+\frac{1}{2}\left[\left(w_{h}\right)_{x}\right]_{j-\frac{1}{2}}^{2}+\frac{1}{2}\left[\left(w_{h}\right)_{x x}\right]_{j-\frac{1}{2}}^{2} \geq 0$. Invoking (5.21) then finishes the proof.

Remark. Our scheme can be generalized to solve equations of the type

$$
\begin{gathered}
u_{t}+b(u)_{x x x}=0, \\
u_{t}+b(u)_{x x x x x}=0, \\
u_{t}+b(u)_{x}+b(u)_{x x x x}=0
\end{gathered}
$$

and so on. The idea is to first get the $L^{2}$ projection of $b(u)$ onto $V_{h}^{k}$ as a function $w_{h}$, then treat the equation as a linear equation as in the case of the $K(n, n, n)$ equation. Denote $B(u)=\int b(u) d u$. If $H$ is positive, we will have a stability for the DG scheme in the form

$$
\frac{d}{d t} \int_{I} B(u) d x \leq 0
$$

\section{Concluding REMARKS AND A PLAN FOR FUtUre WORK}

We have proposed a DG method to solve time dependent PDEs with higher order spatial derivatives. The scheme is formulated by repeated integration by parts of the original equation and then replacing the interface values of the solution by carefully chosen numerical fluxes. Stability and error estimates have been proven for a few representative PDEs. Compared to the LDG method, our DG method has the advantage of easier formulation and implementation, it has a smaller effective stencil (communication is only with immediate neighboring cells regardless of the polynomial degree or the order of the PDE, unlike the LDG method which would have a wider effective stencil for PDEs with higher spatial derivatives), and it may save storage and computational cost as it does not introduce auxiliary variables for the derivatives. Similar to the LDG method, a careful choice of the numerical fluxes is a key ingredient for the stability of the scheme. It turns out that for odd order derivative terms, the choice of the numerical fluxes is similar to that of LDG and is rather straightforward, while for even order derivative terms, extra penalty terms involving the jumps of the solution must be added to the numerical flux to ensure stability, which is different from the LDG choice of fluxes. Other disadvantages against the LDG method include the fact that our DG method is in general inconsistent for lower order $P^{k}$ elements when $k+1$ is smaller than the highest order of the spatial derivative in the PDE, while the LDG method can be designed from $P^{0}$ elements; and the fact that different and sometimes more restrictive nonlinear PDEs can be solved stably by our DG method than by the LDG method. Nevertheless, the new DG method can be designed and proven stable for 
many nonlinear high order PDEs such as the $K(n, n, n)$ equation. Future work will include the development and analysis of this class of DG methods for more general nonlinear PDEs in multi-dimensions, and a more systematic comparison with the LDG method.

\section{A. Appendix: Collections of lemmas And Proofs}

In this appendix, we collect some technical lemmas and proofs for the error estimates.

A.1. A quantity related to the numerical flux. In [29], Zhang and Shu introduced an important quantity to measure the difference between a monotone numerical flux and the physical flux.

Lemma A.1 ([29]). $w \in L^{2}(0,1)$, on each cell boundary point we define

$$
\alpha(\hat{f} ; w) \equiv \alpha\left(\hat{f} ; w^{-}, w^{+}\right) \triangleq \begin{cases}{[w]^{-1}(f(\bar{w})-\hat{f}(w)),} & \text { if }[w] \neq 0, \\ \frac{1}{2}\left|f^{\prime}(\bar{w})\right|, & \text { if }[w]=0,\end{cases}
$$

where $\hat{f}(w) \equiv \hat{f}\left(w^{-}, w^{+}\right)$is a monotone numerical flux consistent with the given flux $f$. Then $\alpha(\hat{f} ; w)$ is non-negative and bounded for any bounded $\left(w^{-}, w^{+}\right) \in \mathbb{R}^{2}$. Moreover, we have

$$
\frac{1}{2}\left|f^{\prime}(\bar{w})\right| \leq \alpha(\hat{f} ; w)+C_{\star}|[w]|, \quad-\frac{1}{8}\left|f^{\prime \prime}(\bar{w})\right|[w] \leq \alpha(\hat{f} ; w)+C_{\star}|[w]|^{2} .
$$

For simplicity, we will use the following notation:

$$
\alpha(\hat{f} ; w)[\phi]^{2}=\sum_{j=1}^{N} \alpha(\hat{f} ; w)_{j+\frac{1}{2}}[\phi]_{j+\frac{1}{2}}^{2} .
$$

A.2. Proof of Theorem 3.2. Without loss of generality, let us assume $\sigma=1$ and prove for the flux choice (3.4). Let $e_{u}=u-u_{h}$ be the error between the numerical and exact solutions. For the nonlinearity of $f(u)$, following the lines of [26], we would like to make an a priori assumption that, for small enough $h$, it holds that

$$
\left\|u-u_{h}\right\| \leq h
$$

and by the interpolation property,

$$
\left\|e_{u}\right\|_{\infty} \leq C h^{\frac{1}{2}} \quad \text { and } \quad\left\|P_{h} u-u_{h}\right\|_{\infty} \leq C h^{\frac{1}{2}} .
$$

This assumption is unnecessary for linear $f$. Since $u$ clearly satisfies (3.2), we can obtain the cell error equation

$$
\begin{aligned}
& \int_{I_{j}}\left(u-u_{h}\right)_{t} v_{h} d x-\int_{I_{j}}\left(f(u)-f\left(u_{h}\right)\right) v_{h x} d x-\int_{I_{j}}\left(u-u_{h}\right)\left(v_{h}\right)_{x x x} d x \\
& +\left(\left(f(u)-\hat{f}\left(u_{h}^{-}, u_{h}^{+}\right)\right) v_{h}^{-}\right)_{j+\frac{1}{2}}-\left(\left(f(u)-\hat{f}\left(u_{h}^{-}, u_{h}^{+}\right)\right) v_{h}^{+}\right)_{j-\frac{1}{2}} \\
(\mathrm{~A} .3) & +\left(\left(u-u_{h}^{+}\right)\left(v_{h}\right)_{x x}^{-}\right)_{j+\frac{1}{2}}-\left(\left(u-u_{h}^{+}\right)\left(v_{h}\right)_{x x}^{+}\right)_{j-\frac{1}{2}}-\left(\left(u_{x}-\left(u_{h}\right)_{x}^{+}\right)\left(v_{h}\right)_{x}^{-}\right)_{j+\frac{1}{2}} \\
& +\left(\left(u_{x}-\left(u_{h}\right)_{x}^{+}\right)\left(v_{h}\right)_{x}^{+}\right)_{j-\frac{1}{2}}+\left(\left(u_{x x}-\left(u_{h}\right)_{x x}^{-}\right) v_{h}^{-}\right)_{j+\frac{1}{2}} \\
& -\left(\left(u_{x x}-\left(u_{h}\right)_{x x}^{-}\right) v_{h}^{+}\right)_{j-\frac{1}{2}}=0
\end{aligned}
$$

for all $v_{h} \in V_{h}^{k}$. 
Define

$$
\begin{aligned}
& \mathcal{B}_{j}\left(u-u_{h} ; v_{h}\right)=\int_{I_{j}}\left(u-u_{h}\right)_{t} v_{h} d x-\int_{I_{j}}\left(u-u_{h}\right)\left(v_{h}\right)_{x x x} d x \\
& +\left(\left(u-u_{h}^{+}\right)\left(v_{h}\right)_{x x}^{-}\right)_{j+\frac{1}{2}}-\left(\left(u-u_{h}^{+}\right)\left(v_{h}\right)_{x x}^{+}\right)_{j-\frac{1}{2}} \\
& -\left(\left(u_{x}-\left(u_{h}\right)_{x}^{+}\right)\left(v_{h}\right)_{x}^{-}\right)_{j+\frac{1}{2}}+\left(\left(u_{x}-\left(u_{h}\right)_{x}^{+}\right)\left(v_{h}\right)_{x}^{+}\right)_{j-\frac{1}{2}} \\
& +\left(\left(u_{x x}-\left(u_{h}\right)_{x x}^{-}\right) v_{h}^{-}\right)_{j+\frac{1}{2}}-\left(\left(u_{x x}-\left(u_{h}\right)_{x x}^{-}\right) v_{h}^{+}\right)_{j-\frac{1}{2}}
\end{aligned}
$$

and

$$
\begin{aligned}
\mathcal{H}_{j}\left(f ; u, u_{h} ; v_{h}\right) & =\int_{I_{j}}\left(f(u)-f\left(u_{h}\right)\right)\left(v_{h}\right)_{x} d x-\left(\left(f(u)-\hat{f}\left(u_{h}^{-}, u_{h}^{+}\right)\right) v_{h}^{-}\right)_{j+\frac{1}{2}} \\
& +\left(\left(f(u)-\hat{f}\left(u_{h}^{-}, u_{h}^{+}\right)\right) v_{h}^{+}\right)_{j-\frac{1}{2}} .
\end{aligned}
$$

Summing over all $j$, the error equation becomes

$$
\sum_{j=1}^{N} \mathcal{B}_{j}\left(u-u_{h} ; v_{h}\right)=\sum_{j=1}^{N} \mathcal{H}_{j}\left(f ; u, u_{h} ; v_{h}\right)
$$

for all $v_{h} \in V_{h}^{k}$.

Since $k \geq 3$, we can choose a projection $P_{h}$ onto $V_{h}^{k}$ such that, for any $u, P_{h} u$ satisfies

$$
\int_{I_{j}} u v_{h} d x=\int_{I_{j}} P_{h} u v_{h} d x
$$

for any $v_{h} \in V_{h}^{k-3}$ and

$$
P_{h} u^{+}=u^{+}, \quad\left(P_{h} u\right)_{x}^{+}=u_{x}^{+}, \quad\left(P_{h} u\right)_{x x}^{-}=u_{x x}^{-}
$$

at all $x_{j+1 / 2}$.

Denote

$$
w_{h}=P_{h} u-u_{h}, \quad w^{e}=P_{h} u-u
$$

and let $v_{h}=w_{h}$ in (A.6) such that we obtain the energy equality

$$
\sum_{j=1}^{N} \mathcal{B}_{j}\left(w_{h}-w^{e} ; w_{h}\right)=\sum_{j=1}^{N} \mathcal{H}_{j}\left(f ; u, u_{h} ; w_{h}\right) .
$$

We first consider the left side of the energy equality (A.8).

Lemma A.2. The following equality holds:

$$
\sum_{j=1}^{N} \mathcal{B}_{j}\left(w_{h}-w^{e} ; w_{h}\right)=\int_{I}\left(w_{h}\right)_{t} w_{h} d x-\int_{I}\left(w_{h}\right)_{t}^{e} w_{h} d x+\sum_{j=1}^{N} \frac{1}{2}\left[\left(w_{h}\right)_{x}\right]_{j+\frac{1}{2}}^{2} .
$$

Proof of Lemma A.2.

$$
\mathcal{B}_{j}\left(w_{h}-w^{e} ; w_{h}\right)=\mathcal{B}_{j}\left(w_{h} ; w_{h}\right)-\mathcal{B}_{j}\left(w^{e} ; w_{h}\right) .
$$

By a similar argument as that in the stability proof (Theorem 3.1), we have

$$
\sum_{j=1}^{N} \mathcal{B}_{j}\left(w_{h} ; w_{h}\right)=\int_{I}\left(w_{h}\right)_{t} w_{h} d x+\sum_{j=1}^{N} \frac{1}{2}\left[\left(w_{h}\right)_{x}\right]_{j+\frac{1}{2}}^{2} .
$$


From the definition of the projection $P_{h}$,

$$
\mathcal{B}_{j}\left(w^{e} ; w_{h}\right)=\int_{I} w_{t}^{e} w_{h} d x .
$$

Thus, by combining (A.11) and (A.12), we obtain (A.9).

Now, consider the right hand side of (A.8), we can rewrite it into the following form:

$$
\begin{aligned}
& \sum_{j=1}^{N} \mathcal{H}_{j}\left(f ; u, u_{h} ; w_{h}\right)=\sum_{j=1}^{N} \int_{I_{j}}\left(f(u)-f\left(u_{h}\right)\right)\left(w_{h}\right)_{x} d x \\
& +\sum_{j=1}^{N}\left(\left(f(u)-f\left(\overline{u_{h}}\right)\right)\left[w_{h}\right]\right)_{j+\frac{1}{2}}+\sum_{j=1}^{N}\left(\left(f\left(\overline{u_{h}}\right)-\hat{f}\right)\left[w_{h}\right]\right)_{j+\frac{1}{2}} .
\end{aligned}
$$

In [26], $\mathrm{Xu}$ and Shu proved the following lemma.

Lemma A.3 ([26]). The following inequality holds:

$$
\sum_{j=1}^{N}\left(\left(f\left(\overline{u_{h}}\right)-\hat{f}\right)\left[w_{h}\right]\right)_{j+\frac{1}{2}} \leq-\frac{3}{4} \alpha\left(\hat{f} ; u_{h}\right)\left[w_{h}\right]^{2}+C h^{2 k+1} .
$$

Proof of Lemma A.3. The proof is exactly the same as that in [26]. Note that although we use a different projection $P_{h}$, the interpolation property still holds.

Lemma A.4 (26]). The following inequality holds:

$$
\begin{aligned}
& \sum_{j=1}^{N} \int_{I_{j}}\left(f(u)-f\left(u_{h}\right)\right)\left(w_{h}\right)_{x} d x+\sum_{j=1}^{N}\left(\left(f(u)-f\left(\overline{u_{h}}\right)\right)\left[w_{h}\right]\right)_{j+\frac{1}{2}} \\
& \leq \frac{1}{2} \alpha\left(\hat{f} ; u_{h}\right)\left[w_{h}\right]^{2}+\left(C+C_{\star}\left(\left\|w_{h}\right\|_{\infty}+h^{-1}\left\|e_{u}\right\|_{\infty}^{2}\right)\right)\left\|w_{h}\right\|^{2} \\
& +\left(C+C_{\star}\left\|e_{u}\right\|_{\infty}^{2}\right) h^{2 k} .
\end{aligned}
$$

Proof of Lemma A.4. The proof follows the same line as that in 26. Note that our bound in this lemma is different from that in [26; the last term in (A.14) is $O\left(h^{2 k}\right)$ while in [26] it is $O\left(h^{2 k+1}\right)$. This is because we use a different projection $P_{h}$, hence in the $\mathcal{T}_{3}$ term (see the proof in [26]) we have an extra term $-\sum_{j=1}^{N} \int_{I_{j}} f^{\prime}\left(\left(u_{h}\right)_{j}\right) w^{e}\left(w_{h}\right)_{x} d x$.

Combining Lemmas A.3 and A.4, we arrive at the following conclusion.

Corollary A.5 ([26]). The following inequality holds:

$$
\begin{aligned}
& \sum_{j=1}^{N} \mathcal{H}_{j}\left(f ; u, u_{h} ; w_{h}\right) \\
& \leq-\frac{1}{4} \alpha\left(\hat{f} ; u_{h}\right)\left[w_{h}\right]^{2}+\left(C+C_{\star}\left(\left\|w_{h}\right\|_{\infty}+h^{-1}\left\|e_{u}\right\|_{\infty}^{2}\right)\right)\left\|w_{h}\right\|^{2} \\
& +\left(C+C_{\star}\left\|e_{u}\right\|_{\infty}^{2}\right) h^{2 k} .
\end{aligned}
$$


Now, combining (A.9) and (A.15), we obtain

$$
\begin{aligned}
& \int_{I}\left(w_{h}\right)_{t} w_{h} d x+\sum_{j=1}^{N} \frac{1}{2}\left[\left(w_{h}\right)_{x}\right]_{j+\frac{1}{2}}^{2}+\frac{1}{4} \alpha\left(\hat{f} ; u_{h}\right)\left[w_{h}\right]^{2} \\
& \leq \int_{I} w_{t}^{e} w_{h} d x+\left(C+C_{\star}\left(\left\|w_{h}\right\|_{\infty}+h^{-1}\left\|e_{u}\right\|_{\infty}^{2}\right)\right)\left\|w_{h}\right\|^{2}+\left(C+C_{\star}\left\|e_{u}\right\|_{\infty}^{2}\right) h^{2 k} .
\end{aligned}
$$

From Young's inequality,

$$
\begin{aligned}
& \int_{I}\left(w_{h}\right)_{t} w_{h} d x+\sum_{j=1}^{N} \frac{1}{2}\left[\left(w_{h}\right)_{x}\right]_{j+\frac{1}{2}}^{2}+\frac{1}{4} \alpha\left(\hat{f} ; u_{h}\right)\left[w_{h}\right]^{2} \\
& \leq\left(C+C_{\star}\left(\left\|w_{h}\right\|_{\infty}+h^{-1}\left\|e_{u}\right\|_{\infty}^{2}\right)\right)\left\|w_{h}\right\|^{2}+\left(C+C_{\star}\left\|e_{u}\right\|_{\infty}^{2}\right) h^{2 k} .
\end{aligned}
$$

Using the results implied by the a priori assumption,

$$
\frac{1}{2} \frac{d}{d t} \int_{0}^{1} w_{h}^{2} d x \leq C\left\|w_{h}\right\|^{2}+C h^{2 k}
$$

Then Theorem 3.2 follows. Finally, the a priori assumption is justified by (3.8). To be more precise, since $k \geq 3$, we can consider $h$ small enough so that $C h^{k}<\frac{1}{2} h$, where $C$ is the constant in (3.8) determined by the final time $T$. Then, if $t^{*}=$ $\sup \left\{t:\left\|u_{h}(t)-u(t)\right\| \leq h\right\}$, we would have $\left\|u_{h}\left(t^{*}\right)-u\left(t^{*}\right)\right\|=h$ by continuity if $t^{*}$ is finite. On the other hand, our proof implies that (3.8) holds for $t \leq t^{*}$, in particular, $\left\|u_{h}\left(t^{*}\right)-u\left(t^{*}\right)\right\| \leq C h^{k}<\frac{1}{2} h$. This is a contradiction if $t^{*}<T$. Hence $t^{*} \geq T$ and our a priori assumption is justified.

Remark. If $f(u)=0$, then $\sum_{j=1}^{N} \mathcal{H}_{j}\left(f ; u, u_{h} ; w_{h}\right)=0$. We can then easily improve the proof to $(k+1)$-th order accuracy.

A.3. Proof of Theorem 3.3. The proof is similar to that for Theorem 3.2, except that we need to use another projection $P_{h}$ that satisfies, for any $u$,

$$
\int_{I_{j}} u v_{h} d x=\int_{I_{j}} P_{h} u v_{h} d x
$$

for any $v_{h} \in V_{h}^{0}$ and

$$
P_{h} u^{+}=u^{+}, \quad\left(P_{h} u\right)_{x x}^{-}=u_{x x}^{-}
$$

at all $x_{j+1 / 2}$. Then,

$$
\begin{aligned}
\sum_{j=1}^{N} \mathcal{B}_{j}\left(w_{h}-w^{e} ; w_{h}\right)= & \int_{I}\left(w_{h}\right)_{t} w_{h} d x-\int_{I} w_{t}^{e} w_{h} d x \\
& +\sum_{j=1}^{N} \frac{1}{2}\left[\left(w_{h}\right)_{x}\right]_{j+\frac{1}{2}}^{2}-\sum_{j=1}^{N}\left(\left(w^{e}\right)_{x}^{+}\left[\left(w_{h}\right)_{x}\right]\right)_{j+\frac{1}{2}} .
\end{aligned}
$$

Corollary A.5 still holds in this case. Thus we have

$$
\begin{aligned}
& \int_{I}\left(w_{h}\right)_{t} w_{h} d x+\sum_{j=1}^{N} \frac{1}{2}\left[\left(w_{h}\right)_{x}\right]_{j+\frac{1}{2}}^{2}+\frac{1}{4} \alpha\left(\hat{f} ; u_{h}\right)\left[w_{h}\right]^{2}-\sum_{j=1}^{N}\left(\left(w^{e}\right)_{x}^{+}\left[\left(w_{h}\right)_{x}\right]\right)_{j+\frac{1}{2}} \\
& \leq \int_{I} w_{t}^{e} w_{h} d x+\left(C+C_{\star}\left(\left\|w_{h}\right\|_{\infty}+h^{-1}\left\|e_{u}\right\|_{\infty}^{2}\right)\right)\left\|w_{h}\right\|^{2}+\left(C+C_{\star}\left\|e_{u}\right\|_{\infty}^{2}\right) h^{4} .
\end{aligned}
$$


From Young's inequality,

$$
\begin{aligned}
& \int_{I}\left(w_{h}\right)_{t} w_{h} d x+\sum_{j=1}^{N} \frac{1}{4}\left[\left(w_{h}\right)_{x}\right]_{j+\frac{1}{2}}^{2}+\frac{1}{4} \alpha\left(\hat{f} ; u_{h}\right)\left[w_{h}\right]^{2} \\
& \leq\left(C+C_{\star}\left(\left\|w_{h}\right\|_{\infty}+h^{-1}\left\|e_{u}\right\|_{\infty}^{2}\right)\right)\left\|w_{h}\right\|^{2}+\left(C+C_{\star}\left\|e_{u}\right\|_{\infty}^{2}\right) h^{4}+C h^{3} .
\end{aligned}
$$

Using the results implied by the a priori assumption,

$$
\frac{1}{2} \frac{d}{d t} \int_{0}^{1} w_{h}^{2} d x \leq C\left\|w_{h}\right\|^{2}+C h^{3} .
$$

Thus, Theorem 3.3 follows. Finally, the a priori assumption is justified by (3.9).

A.4. Proof of Theorem 4.2. Let $e_{u}=u-u_{h}$ be the error between the numerical and exact solutions. For a nonlinear $f(u)$, we will still need the a priori assumption (A.1). This assumption is unnecessary for linear $f$.

Since $u$ clearly satisfies (4.3), we can obtain the cell error equation

$$
\begin{aligned}
& \int_{I_{j}}\left(u-u_{h}\right)_{t} v_{h} d x-\int_{I_{j}}\left(f(u)-f\left(u_{h}\right)\right)\left(v_{h}\right)_{x} d x-\int_{I_{j}}\left(u-u_{h}\right)\left(v_{h}\right)_{x x} d x \\
& +\left(\left(f(u)-\hat{f}\left(u_{h}^{-}, u_{h}^{+}\right)\right) v_{h}^{-}\right)_{j+\frac{1}{2}}-\left(\left(f(u)-\hat{f}\left(u_{h}^{-}, u_{h}^{+}\right)\right) v_{h}^{+}\right)_{j-\frac{1}{2}} \\
& \left.\left.-\left(\left(u-u_{h}\right)_{x}^{-}+\xi\left[u-u_{h}\right]\right) v_{h}^{-}\right)_{j+\frac{1}{2}}+\left(\left(u-u_{h}\right)_{x}^{-}+\xi\left[u-u_{h}\right]\right) v_{h}^{+}\right)_{j-\frac{1}{2}} \\
& +\left(\left(u-u_{h}^{+}\right)\left(v_{h}\right)_{x}^{-}\right)_{j+\frac{1}{2}}-\left(\left(u-u_{h}^{+}\right)\left(v_{h}\right)_{x}^{+}\right)_{j-\frac{1}{2}}=0
\end{aligned}
$$

for all $v_{h} \in V_{h}^{k}$.

Define

$$
\begin{aligned}
& \mathcal{B}_{j}\left(u-u_{h} ; v_{h}\right)=\int_{I_{j}}\left(u-u_{h}\right)_{t} v_{h} d x \\
& \left.\quad-\int_{I_{j}}\left(u-u_{h}\right)\left(v_{h}\right)_{x x} d x-\left(\left(u-u_{h}\right)_{x}^{-}+\xi\left[u-u_{h}\right]\right) v_{h}^{-}\right)_{j+\frac{1}{2}} \\
& \left.\quad+\left(\left(u-u_{h}\right)_{x}^{-}+\xi\left[u-u_{h}\right]\right) v_{h}^{+}\right)_{j-\frac{1}{2}}+\left(\left(u-u_{h}^{+}\right)\left(v_{h}\right)_{x}^{-}\right)_{j+\frac{1}{2}}-\left(\left(u-u_{h}^{+}\right)\left(v_{h}\right)_{x}^{+}\right)_{j-\frac{1}{2}}
\end{aligned}
$$

and

$$
\begin{aligned}
& \mathcal{H}_{j}\left(f ; u, u_{h} ; v_{h}\right)=\int_{I_{j}}\left(f(u)-f\left(u_{h}\right)\right)\left(v_{h}\right)_{x} d x \\
& \quad-\left(\left(f(u)-\hat{f}\left(u_{h}^{-}, u_{h}^{+}\right)\right) v_{h}^{-}\right)_{j+\frac{1}{2}}+\left(\left(f(u)-\hat{f}\left(u_{h}^{-}, u_{h}^{+}\right)\right) v_{h}^{+}\right)_{j-\frac{1}{2}} .
\end{aligned}
$$

Summing over all $j$, the error equation becomes

$$
\sum_{j=1}^{N} \mathcal{B}_{j}\left(u-u_{h} ; v_{h}\right)=\sum_{j=1}^{N} \mathcal{H}_{j}\left(f ; u, u_{h} ; v_{h}\right)
$$

for all $v_{h} \in V_{h}^{k}$.

When $k \geq 2$, we can choose a projection $P_{h}$ onto $V_{h}^{k}$ such that, for any $u, P_{h} u$ satisfies

$$
\int_{I_{j}} u v_{h} d x=\int_{I_{j}} P_{h} u v_{h} d x
$$


for any $v_{h} \in V_{h}^{k-2}$ and

$$
P_{h} u^{+}=u^{+}, \quad\left(P_{h} u\right)_{x}^{-}=u_{x}^{-}
$$

at all $x_{j+1 / 2}$.

Denote

$$
w_{h}=P_{h} u-u_{h}, \quad w^{e}=P_{h} u-u
$$

and let $v_{h}=w_{h}$ in (A.20); we obtain the energy equality

$$
\sum_{j=1}^{N} \mathcal{B}_{j}\left(w_{h}-w^{e} ; w_{h}\right)=\sum_{j=1}^{N} \mathcal{H}_{j}\left(f ; u, u_{h} ; w_{h}\right) .
$$

It is easy to verify the following equality:

$$
\begin{aligned}
& \sum_{j=1}^{N} \mathcal{B}_{j}\left(w_{h}-w^{e} ; w_{h}\right)=\int_{I}\left(w_{h}\right)_{t} w_{h} d x-\int_{I} w_{t}^{e} w_{h} d x \\
& \quad+\int_{I}\left(w_{h}\right)_{x}^{2} d x+\sum_{j=1}^{N}\left(2\left[w_{h}\right]\left(w_{h}\right)_{x}^{-}+\xi\left[w_{h}\right]^{2}\right)_{j+\frac{1}{2}}+\sum_{j=1}^{N} \xi\left(\left(w^{e}\right)^{-}\left[w_{h}\right]\right)_{j+\frac{1}{2}} .
\end{aligned}
$$

Now, consider the right hand side of (A.22). Corollary A.5 will still hold for this projection. We obtain

$$
\begin{aligned}
& \int_{I}\left(w_{h}\right)_{t} w_{h} d x+\int_{I}\left(w_{h}\right)_{x}^{2} d x+\sum_{j=1}^{N}\left(2\left[w_{h}\right]\left(w_{h}\right)_{x}^{-}+\xi\left[w_{h}\right]^{2}\right)_{j+\frac{1}{2}} \\
& +\sum_{j=1}^{N} \xi\left(\left(w^{e}\right)^{-}\left[w_{h}\right]\right)_{j+\frac{1}{2}}+\frac{1}{4} \alpha\left(\hat{f} ; u_{h}\right)\left[w_{h}\right]^{2} \\
& \leq \int_{I} w_{t}^{e} w_{h} d x+\left(C+C_{\star}\left(\left\|w_{h}\right\|_{\infty}+h^{-1}\left\|e_{u}\right\|_{\infty}^{2}\right)\right)\left\|w_{h}\right\|^{2}+\left(C+C_{\star}\left\|e_{u}\right\|_{\infty}^{2}\right) h^{2 k} .
\end{aligned}
$$

From the choice of $\xi$ and the stability proof,

$$
\begin{aligned}
& \int_{I}\left(w_{h}\right)_{t} w_{h} d x+\sum_{j=1}^{N} \frac{C}{h}\left[w_{h}\right]_{j+\frac{1}{2}}^{2}+\sum_{j=1}^{N} \xi\left(\left(w^{e}\right)^{-}\left[w_{h}\right]\right)_{j+\frac{1}{2}}+\frac{1}{4} \alpha\left(\hat{f} ; u_{h}\right)\left[w_{h}\right]^{2} \\
& \leq \int_{I} w_{t}^{e} w_{h} d x+\left(C+C_{\star}\left(\left\|w_{h}\right\|_{\infty}+h^{-1}\left\|e_{u}\right\|_{\infty}^{2}\right)\right)\left\|w_{h}\right\|^{2}+\left(C+C_{\star}\left\|e_{u}\right\|_{\infty}^{2}\right) h^{2 k} .
\end{aligned}
$$

From $\xi=\frac{C}{h}$ and Young's inequality,

$$
\begin{aligned}
& \int_{I}\left(w_{h}\right)_{t} w_{h} d x+\frac{1}{4} \alpha\left(\hat{f} ; u_{h}\right)\left[w_{h}\right]^{2} \\
& \leq\left(C+C_{\star}\left(\left\|w_{h}\right\|_{\infty}+h^{-1}\left\|e_{u}\right\|_{\infty}^{2}\right)\right)\left\|w_{h}\right\|^{2}+\left(C+C_{\star}\left\|e_{u}\right\|_{\infty}^{2}\right) h^{2 k} .
\end{aligned}
$$

Using the results implied by the a priori assumption (A.1),

$$
\frac{1}{2} \frac{d}{d t} \int_{0}^{1} w_{h}^{2} d x \leq C\left\|w_{h}\right\|^{2}+C h^{2 k}
$$

Then Theorem 4.2 follows for this $k \geq 2$ case. Finally, the a priori assumption is justified by (4.10).

For the case of $k=1$, we further assume that the convection term is also linear, namely $f(u)=c u$. This is to avoid the need of the a priori assumption (A.1), 
which is no longer justifiable since our error estimate is of the same order $O(h)$ in this case. The proof of the error estimate is similar to that for the $k \geq 2$ case given above, except that we need to use another projection $P_{h}$ that satisfies, for any $u$,

$$
\int_{I_{j}} u v_{h} d x=\int_{I_{j}} P_{h} u v_{h} d x
$$

for any $v_{h} \in V_{h}^{0}$ and

$$
P_{h} u^{+}=u^{+}
$$

at all $x_{j+1 / 2}$. Then,

$$
\begin{aligned}
& \sum_{j=1}^{N} \mathcal{B}_{j}\left(w_{h}-w^{e} ; w_{h}\right)=\int_{I}\left(w_{h}\right)_{t} w_{h} d x-\int_{I} w_{t}^{e} w_{h} d x+\int_{I}\left(w_{h}\right)_{x}^{2} d x \\
& \quad+\sum_{j=1}^{N}\left(2\left[w_{h}\right]\left(w_{h}\right)_{x}^{-}+\xi\left[w_{h}\right]^{2}\right)_{j+\frac{1}{2}}-\sum_{j=1}^{N}\left(\left(\left(w_{x}^{e}\right)^{-}+\xi\left[w^{e}\right]\right)\left[w_{h}\right]\right)_{j+\frac{1}{2}} .
\end{aligned}
$$

Corollary A.5 still holds in this case. Thus we have

$$
\begin{gathered}
\int_{I}\left(w_{h}\right)_{t} w_{h} d x+\int_{I}\left(w_{h}\right)_{x}^{2} d x+\sum_{j=1}^{N}\left(2\left[w_{h}\right]\left(w_{h}\right)_{x}^{-}+\xi\left[w_{h}\right]^{2}\right)_{j+\frac{1}{2}} \\
-\sum_{j=1}^{N}\left(\left(\left(w_{x}^{e}\right)^{-}+\xi\left[w^{e}\right]\right)\left[w_{h}\right]\right)_{j+\frac{1}{2}}+\frac{1}{4} \alpha\left(\hat{f} ; u_{h}\right)\left[w_{h}\right]^{2} \\
\leq \int_{I} w_{t}^{e} w_{h} d x+C\left\|w_{h}\right\|^{2}+C h^{2}
\end{gathered}
$$

From Young's inequality,

$$
\int_{I}\left(w_{h}\right)_{t} w_{h} d x+\frac{1}{4} \alpha\left(\hat{f} ; u_{h}\right)\left[w_{h}\right]^{2} \leq C|| w_{h} \|^{2}+C h^{2} .
$$

Thus, Theorem 4.2 follows also for this $k=1$ case with a linear convection flux.

A.5. Proof of Theorem 5.2. Without loss of generality, let us assume $\sigma=1$ and prove for the flux choice $\hat{u}_{h}=u_{h}^{+},\left(\tilde{u_{h}}\right)_{x}=\left(u_{h}\right)_{x}^{+},\left(\bar{u}_{h}\right)_{x x}=\left(u_{h}\right)_{x x}^{-},\left(\check{u}_{h}\right)_{x x x}=$ $\left(u_{h}\right)_{x x x}^{-}$and $\left(\dot{u}_{h}\right)_{x x x x}=\left(u_{h}\right)_{x x x x}^{-}$. Let $e_{u}=u-u_{h}$ be the error between the numerical and exact solutions. For a nonlinear $f(u)$, we will still need the a priori assumption (A.1). This assumption is unnecessary for linear $f$.

Because $u$ clearly satisfies (15.2), we can obtain the cell error equation

$$
\begin{aligned}
& \int_{I_{j}}\left(u-u_{h}\right)_{t} v_{h} d x-\int_{I_{j}}\left(f(u)-f\left(u_{h}\right)\right)\left(v_{h}\right)_{x} d x-\int_{I_{j}}\left(u-u_{h}\right)\left(v_{h}\right)_{x x x x x} d x \\
& +\left(\left(f(u)-\hat{f}\left(u_{h}^{-}, u_{h}^{+}\right)\right) v_{h}^{-}\right)_{j+\frac{1}{2}}-\left(\left(f(u)-\hat{f}\left(u_{h}^{-}, u_{h}^{+}\right)\right) v_{h}^{+}\right)_{j-\frac{1}{2}} \\
& +\left(\left(u-u_{h}^{+}\right)\left(v_{h}\right)_{x x x x}^{-}\right)_{j+\frac{1}{2}}-\left(\left(u-u_{h}^{+}\right)\left(v_{h}\right)_{x x x x}^{+}\right)_{j-\frac{1}{2}} \\
& -\left(\left(u_{x}-\left(u_{h}\right)_{x}^{+}\right)\left(v_{h}\right)_{x x x}^{-}\right)_{j+\frac{1}{2}} \\
& +\left(\left(u_{x}-\left(u_{h}\right)_{x}^{+}\right)\left(v_{h}\right)_{x x x}^{+}\right)_{j-\frac{1}{2}}+\left(\left(u_{x x}-\left(u_{h}\right)_{x x}^{-}\right)\left(v_{h}\right)_{x x}^{-}\right)_{j+\frac{1}{2}} \\
& -\left(\left(u_{x x}-\left(u_{h}\right)_{x x}^{-}\right)\left(v_{h}\right)_{x x}^{+}\right)_{j-\frac{1}{2}} \\
& -\left(\left(u_{x x x}-\left(u_{h}\right)_{x x x}^{-}\right)\left(v_{h}\right)_{x}^{-}\right)_{j+\frac{1}{2}}+\left(\left(u_{x x x}-\left(u_{h}\right)_{x x x}^{-}\right)\left(v_{h}\right)_{x}^{+}\right)_{j-\frac{1}{2}} \\
& +\left(\left(u_{x x x x}-\left(u_{h}\right)_{x x x x}^{-}\right)\left(v_{h}\right)^{-}\right)_{j+\frac{1}{2}}-\left(\left(u_{x x x x}-\left(u_{h}\right)_{x x x x}^{-}\right)\left(v_{h}\right)^{+}\right)_{j-\frac{1}{2}}=0
\end{aligned}
$$

for all $v_{h} \in V_{h}^{k}$. 
Define

(A.24)

$$
\begin{aligned}
& \mathcal{B}_{j}\left(u-u_{h} ; v_{h}\right)=\int_{I_{j}}\left(u-u_{h}\right)_{t} v_{h} d x-\int_{I_{j}}\left(u-u_{h}\right)\left(v_{h}\right)_{x x x x x} d x \\
& +\left(\left(u-u_{h}^{+}\right)\left(v_{h}\right)_{x x x x}^{-}\right)_{j+\frac{1}{2}}-\left(\left(u-u_{h}^{+}\right)\left(v_{h}\right)_{x x x x}^{+}\right)_{j-\frac{1}{2}}-\left(\left(u_{x}-\left(u_{h}\right)_{x}^{+}\right)\left(v_{h}\right)_{x x x}^{-}\right)_{j+\frac{1}{2}} \\
& +\left(\left(u_{x}-\left(u_{h}\right)_{x}^{+}\right)\left(v_{h}\right)_{x x x}^{+}\right)_{j-\frac{1}{2}}+\left(\left(u_{x x}-\left(u_{h}\right)_{x x}^{-}\right)\left(v_{h}\right)_{x x}^{-}\right)_{j+\frac{1}{2}} \\
& -\left(\left(u_{x x}-\left(u_{h}\right)_{x x}^{-}\right)\left(v_{h}\right)_{x x}^{+}\right)_{j-\frac{1}{2}} \\
& -\left(\left(u_{x x x}-\left(u_{h}\right)_{x x x}^{-}\right)\left(v_{h}\right)_{x}^{-}\right)_{j+\frac{1}{2}}+\left(\left(u_{x x x}-\left(u_{h}\right)_{x x x}^{-}\right)\left(v_{h}\right)_{x}^{+}\right)_{j-\frac{1}{2}} \\
& +\left(\left(u_{x x x x}-\left(u_{h}\right)_{x x x x}^{-}\right)\left(v_{h}\right)^{-}\right)_{j+\frac{1}{2}}-\left(\left(u_{x x x x}-\left(u_{h}\right)_{x x x x}^{-}\right)\left(v_{h}\right)^{+}\right)_{j-\frac{1}{2}}
\end{aligned}
$$

and

$$
\begin{aligned}
\mathcal{H}_{j}\left(f ; u, u_{h} ; v_{h}\right) & =\int_{I_{j}}\left(f(u)-f\left(u_{h}\right)\right)\left(v_{h}\right)_{x} d x \\
& -\left(\left(f(u)-\hat{f}\left(u_{h}^{-}, u_{h}^{+}\right)\right) v_{h}^{-}\right)_{j+\frac{1}{2}}+\left(\left(f(u)-\hat{f}\left(u_{h}^{-}, u_{h}^{+}\right)\right) v_{h}^{+}\right)_{j-\frac{1}{2}} .
\end{aligned}
$$

Summing over all $j$, the error equation becomes

$$
\sum_{j=1}^{N} \mathcal{B}_{j}\left(u-u_{h} ; v_{h}\right)=\sum_{j=1}^{N} \mathcal{H}_{j}\left(f ; u, u_{h} ; v_{h}\right)
$$

for all $v_{h} \in V_{h}^{k}$.

Since $k \geq 5$, we can choose a projection $P_{h}$ onto $V_{h}^{k}$ such that, for any $u, P_{h} u$ satisfies

$$
\int_{I_{j}} u v_{h} d x=\int_{I_{j}} P_{h} u v_{h} d x
$$

for any $v_{h} \in V_{h}^{k-5}$ and

$$
\begin{gathered}
P_{h} u^{+}=u^{+}, \quad\left(P_{h} u\right)_{x}^{+}=u_{x}^{+}, \quad\left(P_{h} u\right)_{x x}^{-}=u_{x x}^{-}, \\
\left(P_{h} u\right)_{x x x}^{-}=u_{x x x}^{-}, \quad\left(P_{h} u\right)_{x x x x}^{-}=u_{x x x x}^{-}
\end{gathered}
$$

at all $x_{j+1 / 2}$.

Using the same notations as before, we denote

$$
w_{h}=P_{h} u-u_{h}, \quad w^{e}=P_{h} u-u
$$

and let $v_{h}=w_{h}$ in (A.26) to obtain the energy equality

$$
\sum_{j=1}^{N} \mathcal{B}_{j}\left(w_{h}-w^{e} ; w_{h}\right)=\sum_{j=1}^{N} \mathcal{H}_{j}\left(f ; u, u_{h} ; w_{h}\right) .
$$

It is easy to verify the following equality:

$$
\sum_{j=1}^{N} \mathcal{B}_{j}\left(w_{h}-w^{e} ; w_{h}\right)=\int_{I}\left(w_{h}\right)_{t} w_{h} d x-\int_{I} w_{t}^{e} w_{h} d x+\sum_{j=1}^{N} \frac{1}{2}\left[\left(w_{h}\right)_{x x}\right]_{j+\frac{1}{2}}^{2} .
$$


Corollary A.5 still holds for the left hand side of the energy equation, so we obtain

$$
\begin{aligned}
& \int_{I}\left(w_{h}\right)_{t} w_{h} d x+\sum_{j=1}^{N} \frac{1}{2}\left[\left(w_{h}\right)_{x x}\right]_{j+\frac{1}{2}}^{2}+\frac{1}{4} \alpha\left(\hat{f} ; u_{h}\right)\left[w_{h}\right]^{2} \\
& \leq \int_{I} w_{t}^{e} w_{h} d x+\left(C+C_{\star}\left(\left\|w_{h}\right\|_{\infty}+h^{-1}\left\|e_{u}\right\|_{\infty}^{2}\right)\right)\left\|w_{h}\right\|^{2}+\left(C+C_{\star}\left\|e_{u}\right\|_{\infty}^{2}\right) h^{2 k} .
\end{aligned}
$$

By the same reasoning as that in the proof of Theorem 3.2,

$$
\frac{1}{2} \frac{d}{d t} \int_{0}^{1} w_{h}^{2} d x \leq C\left\|w_{h}\right\|^{2}+C h^{2 k}
$$

Finally, the a priori assumption is justified by (5.4).

A.6. Proof of Theorem 5.3. The proof is similar to that for Theorem 5.2, except that we need to use another projection $P_{h}$ which satisfies, for any $u$,

$$
\int_{I_{j}} u v_{h} d x=\int_{I_{j}} P_{h} u v_{h} d x
$$

for any $v_{h} \in V_{h}^{0}$ and

$$
P_{h} u^{+}=u^{+}, \quad\left(P_{h} u\right)_{x}^{+}=u_{x}^{+}, \quad\left(P_{h} u\right)_{x x x}^{-}=u_{x x x}^{-}, \quad\left(P_{h} u\right)_{x x x x}^{-}=u_{x x x x}^{-}
$$

at all $x_{j+1 / 2}$. Then,

$$
\begin{gathered}
\sum_{j=1}^{N} \mathcal{B}_{j}\left(w_{h}-w^{e} ; w_{h}\right)=\int_{I}\left(w_{h}\right)_{t} w_{h} d x-\int_{I} w_{t}^{e} w_{h} d x \\
+\sum_{j=1}^{N} \frac{1}{2}\left[\left(w_{h}\right)_{x x}\right]_{j+\frac{1}{2}}^{2}+\sum_{j=1}^{N}\left(\left(w^{e}\right)_{x x}^{-}\left[\left(w_{h}\right)_{x x}\right]\right)_{j+\frac{1}{2}} .
\end{gathered}
$$

Corollary A.5 still holds in this case. Thus we have

$$
\begin{aligned}
& \int_{I}\left(w_{h}\right)_{t} w_{h} d x+\sum_{j=1}^{N} \frac{1}{2}\left[\left(w_{h}\right)_{x x}\right]_{j+\frac{1}{2}}^{2}+\frac{1}{4} \alpha\left(\hat{f} ; u_{h}\right)\left[w_{h}\right]^{2}+\sum_{j=1}^{N}\left(\left(w^{e}\right)_{x x}^{-}\left[\left(w_{h}\right)_{x x}\right]\right)_{j+\frac{1}{2}} \\
& \leq \int_{I} w_{t}^{e} w_{h} d x+\left(C+C_{\star}\left(\left\|w_{h}\right\|_{\infty}+h^{-1}\left\|e_{u}\right\|_{\infty}^{2}\right)\right)\left\|w_{h}\right\|^{2}+\left(C+C_{\star}\left\|e_{u}\right\|_{\infty}^{2}\right) h^{8} .
\end{aligned}
$$

From Young's inequality,

$$
\begin{aligned}
& \int_{I}\left(w_{h}\right)_{t} w_{h} d x+\sum_{j=1}^{N} \frac{1}{4}\left[\left(w_{h}\right)_{x x}\right]_{j+\frac{1}{2}}^{2}+\frac{1}{4} \alpha\left(\hat{f} ; u_{h}\right)\left[w_{h}\right]^{2} \\
& \leq\left(C+C_{\star}\left(\left\|w_{h}\right\|_{\infty}+h^{-1}\left\|e_{u}\right\|_{\infty}^{2}\right)\right)\left\|w_{h}\right\|^{2}+\left(C+C_{\star}\left\|e_{u}\right\|_{\infty}^{2}\right) h^{8}+C h^{5} .
\end{aligned}
$$

Using the results implied by the a priori assumption,

$$
\frac{1}{2} \frac{d}{d t} \int_{0}^{1} w_{h}^{2} d x \leq C\left\|w_{h}\right\|^{2}+C h^{5} .
$$

Thus, Theorem 5.3 follows. Finally, the a priori assumption is justified by (5.5) . 
A.7. Proof of Theorem 5.5. Without loss of generality, let us assume $\sigma=1$. For a nonlinear $f(u)$, we will still need the a priori assumption (A.1). This assumption is unnecessary for linear $f$. Since $u$ clearly satisfies (5.9), we can obtain the cell error equation

(A.31)

$$
\begin{aligned}
& \int_{I_{j}}\left(u-u_{h}\right)_{t} v_{h} d x-\int_{I_{j}}\left(f(u)-f\left(u_{h}\right)\right)\left(v_{h}\right)_{x} d x+\int_{I_{j}}\left(u-u_{h}\right)\left(v_{h}\right)_{x x x x} d x \\
& +\left(\left(f(u)-\hat{f}\left(u_{h}^{-}, u_{h}^{+}\right)\right) v_{h}^{-}\right)_{j+\frac{1}{2}}-\left(\left(f(u)-\hat{f}\left(u_{h}^{-}, u_{h}^{+}\right)\right) v_{h}^{+}\right)_{j-\frac{1}{2}} \\
& -\left(\left(u-u_{h}^{+}\right)\left(v_{h}\right)_{x x x}^{-}\right)_{j+\frac{1}{2}}+\left(\left(u-u_{h}^{+}\right)\left(v_{h}\right)_{x x x}^{+}\right)_{j-\frac{1}{2}} \\
& +\left(\left(u_{x}-\left(u_{h}\right)_{x}^{-}\right)\left(v_{h}\right)_{x x}^{-}\right)_{j+\frac{1}{2}}-\left(\left(u_{x}-\left(u_{h}\right)_{x}^{-}\right)\left(v_{h}\right)_{x x}^{+}\right)_{j-\frac{1}{2}} \\
& -\left(\left(u_{x x}-\left(u_{h}\right)_{x x}^{+}\right)\left(v_{h}\right)_{x}^{-}\right)_{j+\frac{1}{2}}+\left(\left(u_{x x}-\left(u_{h}\right)_{x x}^{+}\right)\left(v_{h}\right)_{x}^{+}\right)_{j-\frac{1}{2}} \\
& +\left(\left(\left(u-u_{h}\right)_{x x x}^{-}-\xi\left[u-u_{h}\right]\right)\left(v_{h}\right)^{-}\right)_{j+\frac{1}{2}}-\left(\left(\left(u-u_{h}\right)_{x x x}^{-}-\xi\left[u-u_{h}\right]\right)\left(v_{h}\right)^{+}\right)_{j-\frac{1}{2}} \\
& =0
\end{aligned}
$$

for all $v_{h} \in V_{h}^{k}$.

Define

$$
\begin{aligned}
\mathcal{B}_{j}(u & \left.-u_{h} ; v_{h}\right)=\int_{I_{j}}\left(u-u_{h}\right)_{t} v_{h} d x+\int_{I_{j}}\left(u-u_{h}\right)\left(v_{h}\right)_{x x x x} d x \\
& -\left(\left(u-u_{h}^{+}\right)\left(v_{h}\right)_{x x x}^{-}\right)_{j+\frac{1}{2}} \\
& +\left(\left(u-u_{h}^{+}\right)\left(v_{h}\right)_{x x x}^{+}\right)_{j-\frac{1}{2}}+\left(\left(u_{x}-\left(u_{h}\right)_{x}^{-}\right)\left(v_{h}\right)_{x x}^{-}\right)_{j+\frac{1}{2}} \\
& -\left(\left(u_{x}-\left(u_{h}\right)_{x}^{-}\right)\left(v_{h}\right)_{x x}^{+}\right)_{j-\frac{1}{2}}-\left(\left(u_{x x}-\left(u_{h}\right)_{x x}^{+}\right)\left(v_{h}\right)_{x}^{-}\right)_{j+\frac{1}{2}} \\
& +\left(\left(u_{x x}-\left(u_{h}\right)_{x x}^{+}\right)\left(v_{h}\right)_{x}^{+}\right)_{j-\frac{1}{2}}+\left(\left(\left(u-u_{h}\right)_{x x x}^{-}-\xi\left[u-u_{h}\right]\right) v_{h}^{-}\right)_{j+\frac{1}{2}} \\
& -\left(\left(\left(u-u_{h}\right)_{x x x}^{-}-\xi\left[u-u_{h}\right]\right) v_{h}^{+}\right)_{j-\frac{1}{2}}
\end{aligned}
$$

and

$$
\begin{aligned}
& \mathcal{H}_{j}\left(f ; u, u_{h} ; v_{h}\right)=\int_{I_{j}}\left(f(u)-f\left(u_{h}\right)\right)\left(v_{h}\right)_{x} d x \\
& \quad-\left(\left(f(u)-\hat{f}\left(u_{h}^{-}, u_{h}^{+}\right)\right) v_{h}^{-}\right)_{j+\frac{1}{2}}+\left(\left(f(u)-\hat{f}\left(u_{h}^{-}, u_{h}^{+}\right)\right) v_{h}^{+}\right)_{j-\frac{1}{2}} .
\end{aligned}
$$

Summing over all $j$, the error equation becomes

$$
\sum_{j=1}^{N} \mathcal{B}_{j}\left(u-u_{h} ; v_{h}\right)=\sum_{j=1}^{N} \mathcal{H}_{j}\left(f ; u, u_{h} ; v_{h}\right)
$$

for all $v_{h} \in V_{h}^{k}$.

When $k \geq 4$, we can choose a projection $P_{h}$ onto $V_{h}^{k}$ such that, for any $u, P_{h} u$ satisfies

for any $v_{h} \in V_{h}^{k-4}$ and

$$
\int_{I_{j}} u v_{h} d x=\int_{I_{j}} P_{h} u v_{h} d x
$$

$$
P_{h} u^{+}=u^{+}, \quad\left(P_{h} u\right)_{x}^{-}=u_{x}^{-}, \quad\left(P_{h} u\right)_{x x}^{+}=u_{x x}^{+}, \quad\left(P_{h} u\right)_{x x x}^{-}=u_{x x x}^{-}
$$

at all $x_{j+1 / 2}$.

Denoting

$$
w_{h}=P_{h} u-u_{h}, \quad w^{e}=P_{h} u-u
$$


and letting $v_{h}=w_{h}$ in (A.34), we obtain the energy equality

$$
\sum_{j=1}^{N} \mathcal{B}_{j}\left(w_{h}-w^{e} ; w_{h}\right)=\sum_{j=1}^{N} \mathcal{H}_{j}\left(f ; u, u_{h} ; w_{h}\right) .
$$

It is not hard to prove the following equality

$$
\begin{aligned}
& \sum_{j=1}^{N} \mathcal{B}_{j}\left(w_{h}-w^{e} ; w_{h}\right)=\int_{I}\left(w_{h}\right)_{t} w_{h} d x-\int_{I} w_{t}^{e} w_{h} d x+\int_{I}\left(w_{h}\right)_{x x}^{2} d x \\
& +\sum_{j=1}^{N}\left(-2\left[w_{h}\right]\left(w_{h}\right)_{x x x}^{-}+\xi\left[w_{h}\right]^{2}\right)_{j+\frac{1}{2}}+\sum_{j=1}^{N} \xi\left(\left(w^{e}\right)^{-}\left[w_{h}\right]\right)_{j+\frac{1}{2}} .
\end{aligned}
$$

Now, consider the right hand side of (A.36). Corollary A.5 still holds for this projection. We obtain

$$
\begin{aligned}
& \int_{I}\left(w_{h}\right)_{t} w_{h} d x+\int_{I}\left(w_{h}\right)_{x x}^{2} d x+\sum_{j=1}^{N}\left(-2\left[w_{h}\right]\left(w_{h}\right)_{x x x}^{-}+\xi\left[w_{h}\right]^{2}\right)_{j+\frac{1}{2}} \\
& \quad+\sum_{j=1}^{N} \xi\left(\left(w^{e}\right)^{-}\left[w_{h}\right]\right)_{j+\frac{1}{2}}+\frac{1}{4} \alpha\left(\hat{f} ; u_{h}\right)\left[w_{h}\right]^{2} \\
& \leq \int_{I} w_{t}^{e} w_{h} d x+\left(C+C_{\star}\left(\left\|w_{h}\right\|_{\infty}+h^{-1}\left\|e_{u}\right\|_{\infty}^{2}\right)\right)\left\|w_{h}\right\|^{2}+\left(C+C_{\star}\left\|e_{u}\right\|_{\infty}^{2}\right) h^{2 k} .
\end{aligned}
$$

From the choice of $\xi$ and the stability proof,

$$
\begin{aligned}
& \int_{I}\left(w_{h}\right)_{t} w_{h} d x+\sum_{j=1}^{N} \frac{C}{h^{3}}\left[w_{h}\right]_{j+\frac{1}{2}}^{2}+\sum_{j=1}^{N} \xi\left(\left(w^{e}\right)^{-}\left[w_{h}\right]\right)_{j+\frac{1}{2}}+\frac{1}{4} \alpha\left(\hat{f} ; u_{h}\right)\left[w_{h}\right]^{2} \\
& \leq \int_{I} w_{t}^{e} w_{h} d x+\left(C+C_{\star}\left(\left\|w_{h}\right\|_{\infty}+h^{-1}\left\|e_{u}\right\|_{\infty}^{2}\right)\right)\left\|w_{h}\right\|^{2} \\
& \quad+\left(C+C_{\star}\left\|e_{u}\right\|_{\infty}^{2}\right) h^{2 k}+C h^{2 k-2} .
\end{aligned}
$$

From $\xi=\frac{C}{h^{3}}$ and Young's inequality,

$$
\begin{aligned}
& \int_{I}\left(w_{h}\right)_{t} w_{h} d x+\frac{1}{4} \alpha\left(\hat{f} ; u_{h}\right)\left[w_{h}\right]^{2} \\
& \leq\left(C+C_{\star}\left(\left\|w_{h}\right\|_{\infty}+h^{-1}\left\|e_{u}\right\|_{\infty}^{2}\right)\right)\left\|w_{h}\right\|^{2}+\left(C+C_{\star}\left\|e_{u}\right\|_{\infty}^{2}\right) h^{2 k}+C h^{2 k-2} .
\end{aligned}
$$

Using the results implied by the a priori assumption (A.1),

$$
\frac{1}{2} \frac{d}{d t} \int_{0}^{1} w_{h}^{2} d x \leq C\left\|w_{h}\right\|^{2}+C h^{2 k-2} .
$$

Then Theorem 5.5 follows for the $k \geq 4$ case and the $a$ priori assumption is justified by (5.16).

The proof for the $k=3$ case is similar to that for the $k \geq 4$ case above, except that we need to use another projection $P_{h}$ that satisfies, for any $u$,

$$
\int_{I_{j}} u v_{h} d x=\int_{I_{j}} P_{h} u v_{h} d x
$$

for any $v_{h} \in V_{h}^{0}$ and

$$
P_{h} u^{+}=u^{+}, \quad\left(P_{h} u\right)_{x}^{-}=u_{x}^{-}, \quad\left(P_{h} u\right)_{x x}^{+}=u_{x x}^{+}
$$


at all $x_{j+1 / 2}$. Then,

$$
\begin{aligned}
& \sum_{j=1}^{N} \mathcal{B}_{j}\left(w_{h}-w^{e} ; w_{h}\right)=\int_{I}\left(w_{h}\right)_{t} w_{h} d x-\int_{I} w_{t}^{e} w_{h} d x+\int_{I}\left(w_{h}\right)_{x x}^{2} d x \\
& +\sum_{j=1}^{N}\left(-2\left[w_{h}\right]\left(w_{h}\right)_{x x x}^{-}+\xi\left[w_{h}\right]^{2}\right)_{j+\frac{1}{2}}+\sum_{j=1}^{N}\left(\left(\left(w^{e}\right)_{x x x}^{-}+\xi\left(w^{e}\right)^{-}\right)\left[w_{h}\right]\right)_{j+\frac{1}{2}} .
\end{aligned}
$$

Then, plugging in Corollary A.5 and applying Young's inequality, we obtain

$$
\begin{aligned}
& \int_{I}\left(w_{h}\right)_{t} w_{h} d x+\frac{1}{4} \alpha\left(\hat{f} ; u_{h}\right)\left[w_{h}\right]^{2} \\
& \leq\left(C+C_{\star}\left(\left\|w_{h}\right\|_{\infty}+h^{-1}\left\|e_{u}\right\|_{\infty}^{2}\right)\right)\left\|w_{h}\right\|^{2}+\left(C+C_{\star}\left\|e_{u}\right\|_{\infty}^{2}\right) h^{6}+C h^{4} .
\end{aligned}
$$

Using the results implied by the a priori assumption,

$$
\frac{1}{2} \frac{d}{d t} \int_{0}^{1} w_{h}^{2} d x \leq C|| w_{h} \|^{2}+C h^{4} .
$$

Thus, Theorem 5.5 follows for the $k=3$ case. Finally, the a priori assumption is justified by (5.16).

\section{REFERENCES}

1. S. Adjerid and H. Temimi, A discontinuous Galerkin method for higher-order ordinary differential equations, Computer Methods in Applied Mechanics and Engineering, to appear.

2. D. Arnold, An interior penalty finite element method with discontinuous elements, SIAM Journal on Numerical Analysis, 19 (1982), pp.742-760. MR664882 (83f:65173)

3. D. Arnold, F. Brezzi, B. Cockburn and L. Marini, Unified analysis of discontinuous Galerkin methods for elliptic problems, SIAM Journal on Numerical Analysis, 39 (2002), pp.1749-1779. MR 1885715 (2002k:65183)

4. G. Baker, Finite element methods for elliptic equations using nonconforming elements, Mathematics of Computation, 31 (1977), pp.45-59. MR0431742 (55:4737)

5. F. Bassi and S. Rebay, A high-order accurate discontinuous finite element method for the numerical solution of the compressible Navier-Stokes equations, Journal of Computational Physics, 131 (1997), pp.267-279. MR.1433934 (97m:76078)

6. C.E. Baumann and J.T. Oden, A discontinuous $h p$ finite element method for convectiondiffusion problems, Computer Methods in Applied Mechanics and Engineering, 175 (1999), pp.311-341. MR.1702201(2000d:65171)

7. P. Ciarlet, The Finite Element Method for Elliptic Problems, North Holland, 1975. MR 0520174 (58:25001)

8. B. Cockburn, S. Hou and C.-W. Shu, The Runge-Kutta local projection discontinuous Galerkin finite element method for conservation laws IV: the multidimensional case, Mathematics of Computation, 54 (1990), pp.545-581. MR.1010597(90k:65162)

9. B. Cockburn, S.-Y. Lin and C.-W. Shu, TVB Runge-Kutta local projection discontinuous Galerkin finite element method for conservation laws III: one dimensional systems, Journal of Computational Physics, 84 (1989), pp.90-113. MR.1015355 (90k:65161)

10. B. Cockburn and C.-W. Shu, TVB Runge-Kutta local projection discontinuous Galerkin finite element method for conservation laws II: general framework, Mathematics of Computation, 52 (1989), pp.411-435. MR983311 (90k:65160)

11. B. Cockburn and C.-W. Shu, The Runge-Kutta local projection P1-discontinuous Galerkin finite element method for scalar conservation laws, Mathematical Modelling and Numerical Analysis, 25 (1991), pp.337-361. MR.1103092 (92e:65128)

12. B. Cockburn and C.-W. Shu, The Runge-Kutta discontinuous Galerkin method for conservation laws V: multidimensional systems, Journal of Computational Physics, 141 (1998), pp.199-224. MR1619652 (99c:65181) 
13. B. Cockburn and C.-W. Shu, The local discontinuous Galerkin method for time-dependent convection-diffusion systems, SIAM Journal on Numerical Analysis, 35 (1998), pp.2440-2463. MR.1655854 (99j:65163)

14. S.M. Cox and P.C. Matthews, Exponential time differencing for stiff systems, Journal of Computational Physics, 176 (2002), pp.430-455. MR.1894772(2003b:65064)

15. G. Gassner, F. Lörcher and C.-D. Munz, A contribution to the construction of diffusion fluxes for finite volume and discontinuous Galerkin schemes, Journal of Computational Physics, 224 (2007), pp. 1049-1063.

16. G. Jiang and C.-W. Shu, On cell entropy inequality for discontinuous Galerkin methods, Mathematics of Computation, 62 (1994), pp.531-538. MR.1223232 (94h:65099)

17. D. Levy, C.-W. Shu and J. Yan, Local discontinuous Galerkin methods for nonlinear dispersive equations, Journal of Computational Physics, 196 (2004), pp.751-772. MR2054354 (2005d:65168)

18. H.-L. Liu and J. Yan, A local discontinuous Galerkin method for the Korteweg-de Vries equation with boundary effect, Journal of Computational Physics, 215 (2006), pp.197-218. MR.2215655 (2006j:65286)

19. J.T. Oden, I. Babuvska and C.E. Baumann, A discontinuous hp finite element method for diffusion problems, Journal of Computational Physics, 146 (1998), pp.491-519. MR1654911 (99m:65173)

20. C.-W. Shu and S. Osher, Efficient implementation of essentially non-oscillatory shockcapturing schemes, Journal of Computational Physics, 77 (1988), pp.439-471. MR954915 (89g:65113)

21. B. van Leer and S. Nomura, Discontinuous Galerkin for diffusion, 17th AIAA Computational Fluid Dynamics Conference (June 6-9, 2005), AIAA paper 2005-5108.

22. Y. Xu and C.-W. Shu, Local discontinuous Galerkin methods for three classes of nonlinear wave equations, Journal of Computational Mathematics, 22 (2004), pp.250-274. MR2058936 (2005h:65175)

23. Y. Xu and C.-W. Shu, Local discontinuous Galerkin methods for nonlinear Schrödinger equations, Journal of Computational Physics, 205 (2005), pp.72-97. MR2132303 (2005m:65228)

24. Y. Xu and C.-W. Shu, Local discontinuous Galerkin methods for two classes of two dimensional nonlinear wave equations, Physica D, 208 (2005), pp.21-58. MR.2167906 (2006f:65100)

25. Y. Xu and C.-W. Shu, Local discontinuous Galerkin methods for the Kuramoto-Sivashinsky equations and the Ito-type coupled KdV equations, Computer Methods in Applied Mechanics and Engineering, 195 (2006), pp.3430-3447. MR2220926 (2006k:65270)

26. Y. Xu and C.-W. Shu, Error estimates of the semi-discrete local discontinuous Galerkin method for nonlinear convection-diffusion and KdV equations, Computer Methods in Applied Mechanics and Engineering, 196 (2007), pp. 3805-3822.

27. J. Yan and C.-W. Shu, A local discontinuous Galerkin method for KdV type equations, SIAM Journal on Numerical Analysis, 40 (2002), pp.769-791. MR.1921677 (2003e:65181)

28. J. Yan and C.-W. Shu, Local discontinuous Galerkin methods for partial differential equations with higher order derivatives, Journal of Scientific Computing, 17 (2002), pp.27-47. MR.1910550

29. Q. Zhang and C.-W. Shu, Error estimates to smooth solutions of Runge-Kutta discontinuous Galerkin methods for scalar conservation laws, SIAM Journal on Numerical Analysis, 42 (2004), pp.641-666. MR2084230(2005h:65149)

Division of Applied Mathematics, Brown University, Providence, Rhode Island 02912 E-mail address: ycheng@dam.brown.edu

Division of Applied Mathematics, Brown University, Providence, Rhode Island 02912 E-mail address: shu@dam.brown.edu 